

\title{
Plasma-enhanced catalysis for the upgrading of methane: A review of modelling and simulation methods
}

Received 00th January 20xx, Accepted 00th January 20xx

DOI: $10.1039 / \times 0 \times x 00000 x$

\author{
Pierre-André Maitre, Matthew S. Bieniek, Panagiotis N. Kechagiopoulos*
}

\begin{abstract}
The direct upgrading of methane to higher hydrocarbons via plasma-catalysis has received particular attention due to the high valorisation potential of the molecule and methane's wide availability in both fossil and sustainable sources. Extensive experimental work has focused on the study of the performance of plasma-catalytic systems. However, it is recognised that most plasma-surface interactions are still not fully understood, and that the combination of experimental, theoretical and modelling studies is essential to gain a fundamental mechanistic insight, leading to advancements in the field. The current review presents the state-of-the-art of simulation methodologies for plasma-phase kinetics, plasma fluid modelling and plasma-catalyst interactions as these have been applied for methane plasmas. Focus is placed on the species that should be considered during modelling, ranging from stable molecules and radicals to ions and excited states. The types of plasmachemical processes these species participate in are discussed and the methods for calculating their rates are presented. Modelling approaches spanning from spatially homogeneous and computationally inexpensive zero-dimensional codes to more complex fluid modelling approaches are covered. First principles calculations concerning plasma relevant species interactions with metal surfaces are finally summarised.
\end{abstract}

\section{Introduction}

Since the first applications of plasma discharges to methane in the presence of a catalyst in the late 90 's, ${ }^{1-4}$ research focus has primarily been placed either on the upgrading of methane into higher hydrocarbons, ${ }^{5-9}$ or its reforming into synthesis gas. ${ }^{10-13}$ Originally, the use of thermal plasmas was dominant, ${ }^{10,14-16}$ however the interest in non-thermal plasmas (NTP) has risen substantially with time. Indeed, the combination of nonthermal plasma with catalysis is a very promising technology, enabling catalysts to operate at low temperatures. The strong non-equilibrium character of non-thermal plasmas has been shown to allow even thermodynamically unfavourable reactions to occur under ambient conditions. ${ }^{17}$ Moreover, in certain cases synergistic effects have been experimentally demonstrated i.e., the performance achieved with plasmacatalysis was higher than the sum of plasma-alone and catalysisalone. ${ }^{18}$ The upgrading of methane via plasma-catalysis has been investigated for the past two decades and has already been proven to be a feasible process. During this period, a considerable amount of experimental work has attempted to probe the effect of input parameters in the performance of plasma-catalytic systems applying conventional end-of-pipe product analysis. Advanced in situ spectroscopic methods have also been applied to elucidate mechanistic details.

The substantial body of experimental work in this field has already been summarised in a variety of reviews. Chen et al.

Chemical and Materials Engineering Group, School of Engineering, University of Aberdeen, Aberdeen, AB24 3UE, UK. E-mail: p.kechagiopoulos@abdn.ac.uk were the first to provide an elaborate overview of research developments in relation to hydrocarbons, including methane, reforming towards hydrogen production. The benefits of single and two-stage plasma-catalysis processes were discussed for non-thermal and thermal plasmas, respectively, while ideal features of power supplies to enhance the efficiency of nonthermal plasmas were proposed. Nozaki and Okazaki later reported on the main principles of non-thermal plasma catalysis of methane ${ }^{17}$ providing an overview of, primarily, prior work of the authors. They concluded that the highest energy efficiencies are achievable at bulk gas temperatures of $400^{\circ} \mathrm{C}$ to $600^{\circ} \mathrm{C}$, where the plasma-generated radical species effectively induce chain propagation reactions. More recently, Scapinello et al. ${ }^{19}$ presented a wide overview of the experimental work on the plasma-enhanced non-oxidative methane reforming providing a thorough account of the different plasma discharges used to date (DBD, corona and spark, gliding arcs, microwave, etc.). Arc thermal plasmas and non-thermal pulsed discharges were identified as the most energy efficient at atmospheric pressure whereas at lower pressures microwave plasmas were discussed to be preferable. Lastly, Puliyalil et al. reviewed systematically the plasma-catalytic conversion of carbon dioxide and methane towards value-added chemicals ${ }^{20}$ and assessed the economic viability and industrialization of the plasma assisted process. Evidently, the low energy efficiency of the plasma-based processes has been identified as the main drawback of the latter in current literature. SriBala et al. ${ }^{21}$ reported that high energy plasmas are more efficient at atmospheric pressures with energy costs spanning from $1.35 \mathrm{MJ} / \mathrm{mol}_{\mathrm{CH} 4}$ for high energydensity spark plasma discharge (the main product being acetylene) to $5.5 \mathrm{MJ} / \mathrm{mol}_{\mathrm{CH} 4}$ for low energy-density DBD (the 
main product being ethane). Atmospheric pressure operation at limited gas inlet flows has also been raised as an issue, for which to be overcome Puliyalil et al. ${ }^{20}$ proposed the use of multitubular reactors according to those used for industrial scale ozone production. ${ }^{22}$

Notwithstanding these issues, the biggest challenge that is faced by all previous research is the lack of insights on the underlying mechanisms of such a complex system. Indeed, the catalyst is responsible for local electric field enhancement, micro discharge formation in pores, and modification of the discharge mode, while the plasma affects the catalyst by increasing the adsorption probability of species, changing the catalyst surface area, and potentially lowering the activation barrier, among many possible interactions. ${ }^{23}$ All these effects make plasma-catalyst systems strongly coupled and highly complex to unravel, thus difficult to design. Given this, experimental studies have typically focused on kinetics and catalysts evaluation utilising simple packed-bed reactors. ${ }^{24-27}$

Fundamental kinetic modelling can provide detailed mechanistic information, without requiring substantial amounts of input data, hence can greatly assist in the analysis of such systems. Due to the complex and dynamic nature of plasma, the kinetic schemes used for its description usually need to account for many different species of different types. The latter can include charged species (anions, cations, and electrons), free radicals, stable and metastable molecules, excited states, all reacting through numerous processes. Using this type of detailed schemes has been employed for different gas discharges and different methane upgrading applications, ranging from oxidative 28-35 $^{28}$ non-oxidative ${ }^{5,36-46}$ and methane conversion in the presence of nitrogen. ${ }^{47-50}$ Besides upgrading, methane plasma kinetic schemes have been employed for other purposes too, with the oldest occurrences being related to plasma-enhanced chemical vapour deposition, ${ }^{51-64}$ and combustion and pyrolysis studies. ${ }^{65-86}$ When reviewing these sources, it is clear that within each field similar techniques have been used but in different ways e.g. by placing the focus on species that were not considered by others or by treating some phenomenon, such as interactions with walls or relaxation and quenching of excited species, in more detail.

The aim of this review is to give an overview of the kinetic modelling approaches in relation to methane plasmas as these have been applied in different fields. A summary of fluid modelling methodologies commonly applied in conjunction with these kinetic models is also provided. Section 2 of the review focuses on the species that have been considered in the literature so far, providing (if existing) experimental evidence on their relevance. Section 3 focuses on the reactive processes that these species participate at, and the methods used to estimate their rate constants if no experimental measurements are available. Section 4 addresses the modelling approaches of methane plasmas (zero-dimensional, fluid modelling, etc.) and the types of discharges these have been applied for, while Section 5 covers the limited work to date on plasma-catalysis modelling and the insights first principles calculations have provided on the interactions of metal surfaces with species relevant to methane plasmas. The review concludes with considerations on areas of interest that would warrant future development.

DOI: 10.1039/DORE00024H

\section{Considered species in reaction mechanisms}

One of plasma's distinctive characteristics is the large number of types of species it comprises. For non-thermal plasmas (i.e. when the electrons' temperature is much higher than that of the bulk gas species so that the plasma can be considered to be far from equilibrium) it is common that all the different species are populated on densities of different orders of magnitude depending on their nature. The types of species considered in methane plasma reaction mechanisms are described in the following.

\subsection{Electrons}

The electrons are the cornerstone species of reactive plasmas and should always be considered in plasma kinetic models. The activation of a gas occurs first by the acceleration of the naturally present free electrons, resulting in their collision with larger species. Upon collision, part of the kinetic energy of the electrons is transmitted to the collision partners. If the transmitted energy is beyond the ionisation threshold, the collision will result in the creation of an ion and an additional free electron. The more the electrons collide, the more they are produced, leading to the so-called electron avalanche phenomenon. The avalanche is generally the precursor to various self-sustainment conditions and transient behaviours that lead to various discharge patterns (filamentary, corona, glow, streamers, etc.). Besides ionisation, the electron collisions are also responsible for other plasma-chemical reactions, such as dissociation and excitation that produce further reactive species, namely radicals and excited states of atoms and molecules, as described in later sections.

In atmospheric and non-thermal applications, the electron temperature generally ranges between 1 to $5 \mathrm{eV}$ for electron densities from $10^{9}$ to $10^{14} \mathrm{~cm}^{-3}$. As the electrons are not in equilibrium with the gas, it is required to account for the variation in the energy of their population. This is described by means of the electron energy distribution function (EEDF), $f(\varepsilon)$, which corresponds to the probability of an electron having the energy $\varepsilon$. The EEDF depends strongly on the electric field and the gas composition of the plasma. Its consideration is of primary importance for the calculation of the rate constants of electron collisions (see Section 3.1).

The EEDF can be assumed a priori to follow specific functions. In the early literature related to methane plasmas some sources used a Maxwellian distribution function. ${ }^{55,87}$ In this case, electrons are considered in thermodynamic equilibrium among each other with elastic collisions dominating. However, this is valid only at high ionisation degrees. Around the same time, Masi et al. used a Druyvesteyn distribution function, ${ }^{54}$ in which the electron energy decreases much faster than the Maxwellian. The Druyvesteyn function was later recommended by Fridman for non-thermal plasmas, where the ionisation degree is low and inelastic collisions of electrons with heavy 
particles cause the EEDF to drop at higher electron energies. ${ }^{88}$ However, the electron distribution function of a particular plasma for a given field can also be obtained by solving numerically the electron Boltzmann equation. This is generally carried out using the so-called two-term approximation, which, although well known in the field of plasma-physics, is not trivial to implement for non-specialists. Nonetheless, nowadays solvers embedded within commercial software (e.g. COMSOL and PLASIMO) or distributed as freeware (BOLSIG $+{ }^{89}$ EEDF, 90 and LoKI $^{91}$ ) are available and are used routinely by the research community, ${ }^{28,37,92}$ so that the assumption of an ideal theoretical distribution, such as that of Maxwell or Druyvesteyn, is no longer required. Figure 1 compares the Maxwell and Druyvesteyn electron energy distribution functions for a pure methane plasma with those obtained using different freely available Boltzmann equation solvers at a mean electron energy of $5 \mathrm{eV}$. Indeed, at these conditions the Druyvesteyn function approximates more closely the results obtained from the more rigorous Boltzmann solvers.

These Boltzmann equation solvers can be used as standalone tools to estimate the rate coefficients of the electron collisions over a range of electric fields, which are then used by kinetic solvers usually via look-up tables. For reactive processes, though, where the gas composition is varying significantly during the timescales simulated, it is advisable that such Boltzmann solvers are used within the plasma kinetic solver, so that the EEDF and the rate constants of electron collisions are updated in response to the changing gas composition.

\subsection{Ions}

The positive ions are the species originating from the ionisation processes that are also responsible for the creation of free electrons. Consequently, their number density is usually equal or about the same to that of the electrons. This is particularly true for electro-positive gases, as in the case of $\mathrm{CH}_{4}$, but this is not always the case for gases such as $\mathrm{O}_{2}, \mathrm{Cl}_{2}$ and $\mathrm{SF}_{6}$ that are

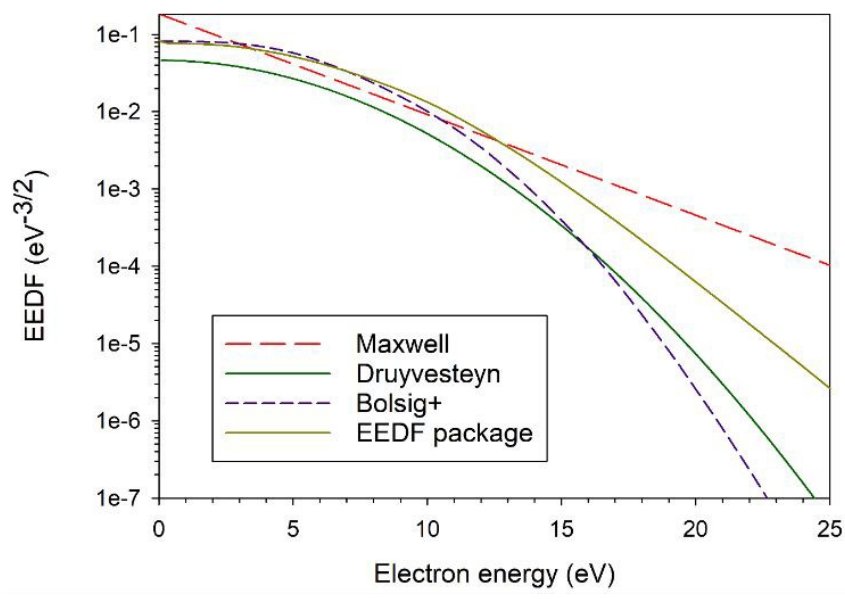

Figure 1. Electron energy distribution in a pure methane plasma for an average electron temperature of $5 \mathrm{eV}$, obtained with ideal Maxwell and Druyvesteyn models and different two-term approximation Boltzmann equation solvers. highly electro-negative and where negative ions arencreated lat significant amounts. ${ }^{93-95}$

DOI: $10.1039 /$ DORE00024H

Nozaki et al. ${ }^{43}$ accounted for the 4 most populated positive ions $\mathrm{CH}_{4}^{+}, \mathrm{CH}_{3}^{+}, \mathrm{CH}_{5}^{+}$and $\mathrm{C}_{2} \mathrm{H}_{5}^{+}$, while modelling steam reforming of methane in dielectric barrier discharges (DBD) in a packed bed reactor, using a simplified version of the kinetic scheme of Tachibana et al. ${ }^{52}$ The latter was initially developed for chemical vapour deposition and included 18 positive ions ranging from $\mathrm{C}^{+}$to $\mathrm{C}_{4} \mathrm{H}_{9}^{+}$. For methane plasmas, it is usually common in literature to not account for negative ions due to the electropositive nature of the plasma. Nonetheless, the dissociative attachment of electrons and methane, leading to the production of negative ions $\mathrm{H}^{-}$and $\mathrm{CH}_{2}^{-}$has been observed experimentally and cross-sections for these collisions were presented by Davies et al. ${ }^{96}$ Later on, Gogolides et al. employed these cross-sections into a model of a well-mixed low pressure RF methane plasma reactor and estimated, in line with above observations, the density of anions to be more than an order of magnitude lower than that of cations depending of pressure (Figure 2). 87,97

When the plasma-assisted activation of methane is carried out in the presence of an oxidant, the negative ions must be included due to the electro-negative nature of oxygen. During modelling of the dry reforming of methane in a dielectric barrier discharge reactor that was assumed to follow the ideal batch model, Snoeckx et al. included the $\mathrm{O}^{-}$and $\mathrm{OH}^{-}$ions, ${ }^{29}$ while De Bie et al. considered 6 negative ions in their model describing the conversion of methane into syngas and oxygenates. ${ }^{32}$ Both works showed that the $\mathrm{O}^{-}$and $\mathrm{OH}^{-}$ions can reach comparable number densities to those of the positive ions and even have a significant impact on the formation of some final products such as methanol. ${ }^{29}$

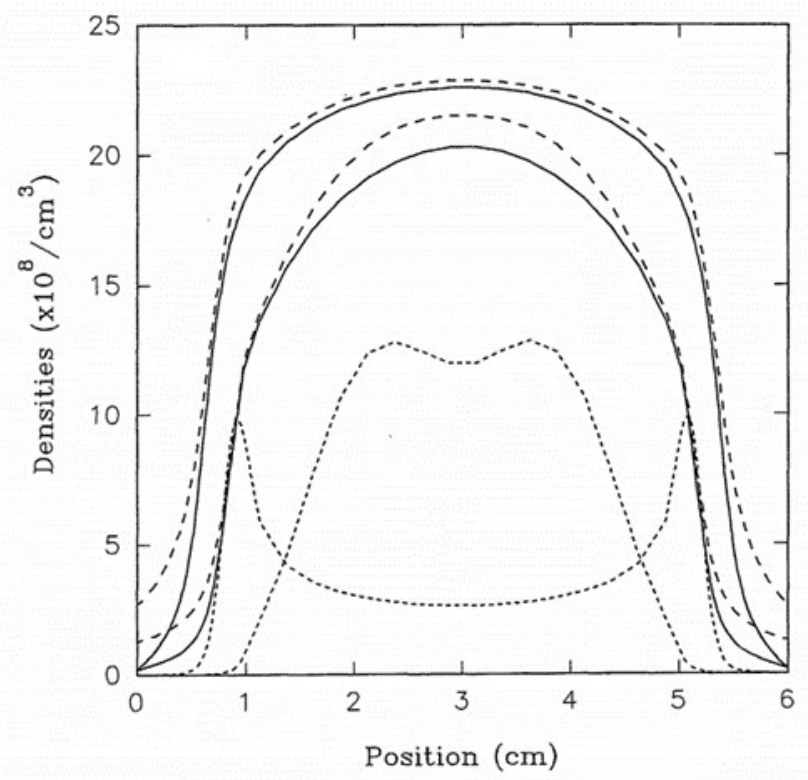

Figure 2. Time-averaged densities of electrons (solid line), positive ions (long dash) and negative ions multiplied by 10 (short dash) in a RF methane plasma across the discharge gap at 300 mTorr (outermost and uppermost curves) and 80 mTorr (innermost and lower lying curves). Reprinted with permission from Gogolides et al. ${ }^{97}$ Copyright (1995) The Physical Society of Japan and The Japan Society of Applied Physics 
Ions are also important species to consider in models related to plasma-surface applications, namely for thin-film deposition during semiconductors processing. In these processes, the ions drift towards the walls of the cell due to the field's influence, where they can incorporate into the substrate, generally with a probability assumed to be $1 .{ }^{55,60}$ Bera et al. ${ }^{61}$ and Farouk et al. ${ }^{98}$ accounted for ion induced stitching reactions, where the ion gets incorporated in the carbon layer by collision with an already-adsorbed specie (typically a radical such as $\mathrm{CH}_{3}$ ) whilst releasing a hydrogen molecule in the bulk at a certain, ion energy dependent, stitching probability.

Finally, in certain studies ions have also been excluded from the total mass balance since, due to their low densities, they represent only a very small fraction of the conversion. In these cases, the electrons are maintained in the kinetic scheme as they are still considered to be the species initiating the dissociation of the reactive gas. For instance, Zhang et al. removed the ions from the plasma chemistry but still included the cross-sections of the respective electron collisions leading to ion creation so as to estimate more accurately the EEDF. ${ }^{47}$ In Legrand et al. the electron density and their temperature were assumed to decay exponentially, a behaviour inspired by their experimental measurements. ${ }^{50}$

\subsection{Reactive molecules}

The considered molecules generally correspond to the reactants and the final products that are expected for a given application, typically those that are observed experimentally. For non-oxidative conversion of methane the most important molecules to account for are $\mathrm{CH}_{4}, \mathrm{H}_{2}$ and $\mathrm{C}_{2}$ hydrocarbons as has been carried out in various works. ${ }^{38,39,43,44,84}$ In some studies $C_{3}$ and higher hydrocarbons have also been included, but their number density, and their respective importance, decreases with the length of the hydrocarbon chain. ${ }^{36,37,40}$ In modelling works, it is common to see that ethane is the product that forms first via methyl coupling, as these radicals are among the most populated and long-lived ones. Ethane creation leads to its subsequent de-hydrogenation towards ethylene and acetylene (see Figure 3). Moreover, ethane leads to the creation of $\mathrm{C}_{2} \mathrm{H}_{5}$

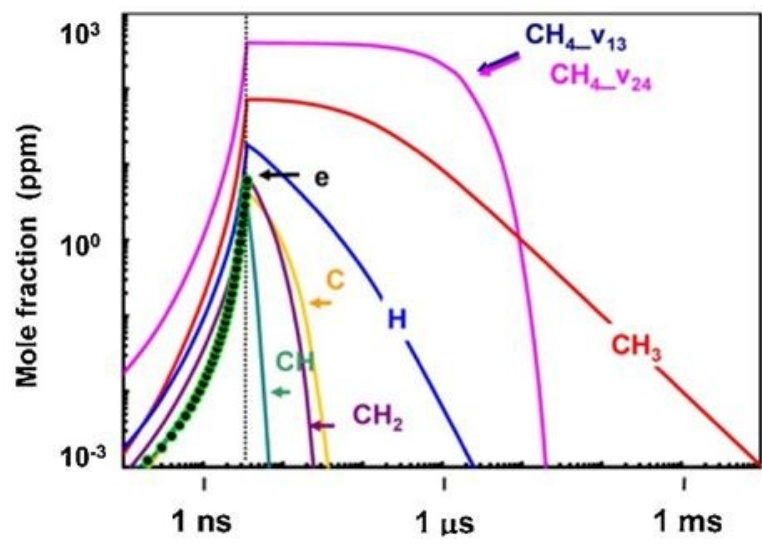

Radical species radicals that are the key species towards the formation of $C_{3} H_{1}$

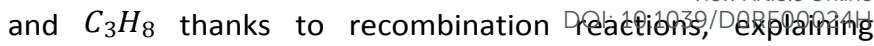
successive production of $C_{3}$ to that of ethane (see Figure 4).

For oxidative conversion, several oxygen-containing species must be included for an accurate description of the chemistry. De Bie et al. for $\mathrm{CH}_{4} / \mathrm{O}_{2}$ and $\mathrm{CH}_{4} / \mathrm{CO}_{2}$ mixtures in a well-mixed DBD reactor considered 22 molecules including 13 oxidized species, such as aldehydes, alcohols and carbon oxides, overall in line with the range of species accounted for in cases of conventional and plasma-assisted combustion studies. ${ }^{32}$ In parallel, Adamovich et al. presented a kinetic scheme for a methane-air plasma in repetitive nanosecond pulse discharges in a perfectly mixed reactor that accounted for the creation of $N$ $O_{x}{ }^{66,67}$ Finally, in the very few cases where nitrogen is introduced (as a co-reactant or as an impurity), nitrogencontaining species such as hydrogen cyanide $(H C N)$, ammonia ( $\left.\mathrm{NH}_{3}\right)$, diazene $\left(\mathrm{N}_{2} \mathrm{H}_{2}\right)$ and hydrazine $\left(\mathrm{N}_{2} \mathrm{H}_{4}\right)$ have also been considered. ${ }^{47-50}$

\subsection{Radicals}

The radicals are the main species leading to the creation of the stable end-products via coupling and chain propagation reactions (see Section 3.2.1) and must, hence, be included in accordance to the molecules considered. Radicals are highly reactive species due to the electron vacancies they contain. Their number densities span across very wide orders of magnitude due to the high variation in their reactivity. Several modelling studies identified $\mathrm{H}, \mathrm{CH}_{3}, \mathrm{CH}_{2}, \mathrm{C}_{2} \mathrm{H}_{3}$ and $\mathrm{C}_{2} \mathrm{H}_{5}$ radicals to be the most abundant ones for non-oxidative applications of methane $37,44,53,72$ with densities that can reach $10^{15} \mathrm{~cm}^{-3}$ (for an atmospheric, room temperature plasma, the density of the background gas is approximately $10^{19} \mathrm{~cm}^{-3}$ ). Assessing the presence of radicals quantitatively during experiments is not a trivial task but has been achieved via isotope analysis, as performed by Scapinello et al. ${ }^{99}$ The authors confirmed the importance of the previously mentioned radicals, measuring number densities in the order of $10^{18}-10^{19} \mathrm{~cm}^{-3}$. The difference in magnitude between these experimental and the previously mentioned modelling results could be attributed

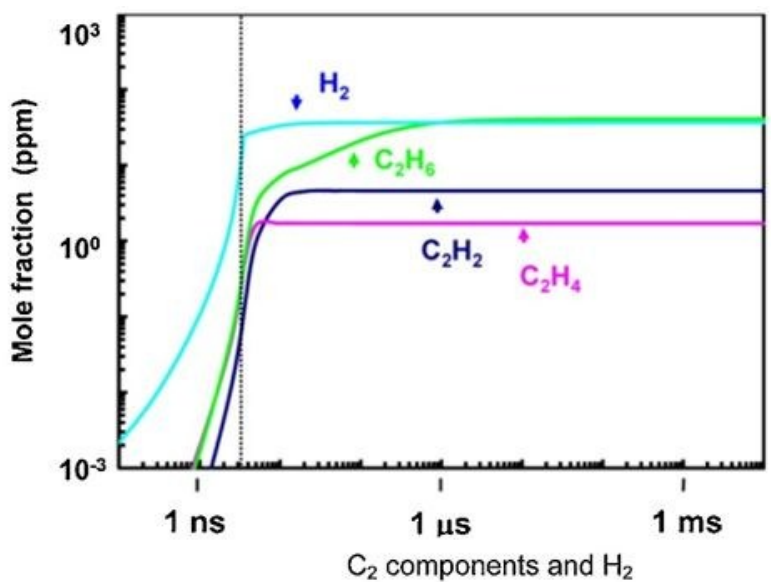

Figure 3. Evolution of radicals and final products after an electron pulse ( $200 \mathrm{Td}, 101.3 \mathrm{kPa}, 300 \mathrm{~K})$ in a pure methane plasma. Reprinted with permission from Nozaki et al. ${ }^{17}$ Copyright (2013) Elsevier 


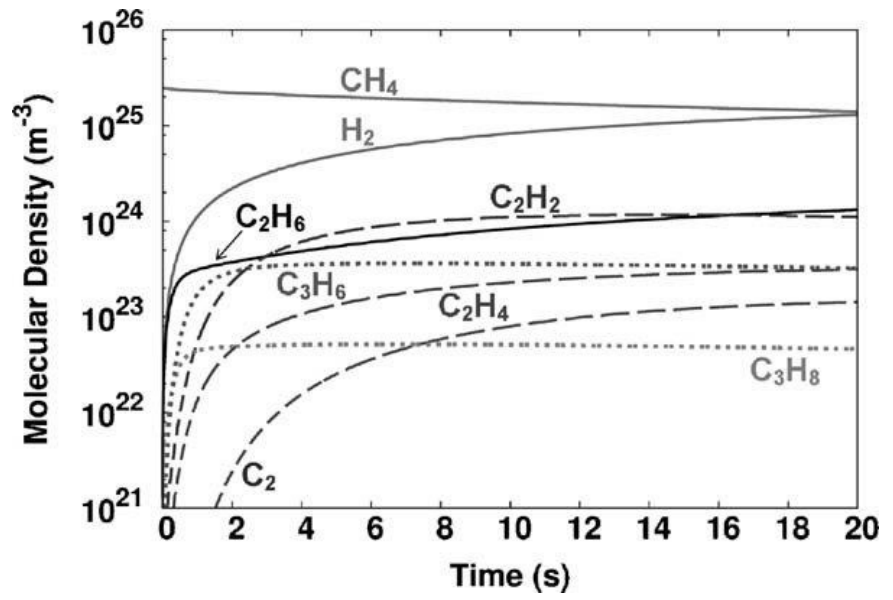

Figure 4. Main molecules densities in a pure methane DBD simulation (6 kV, $10 \mathrm{kHz}$ ). Reprinted with permission from De Bie et al. ${ }^{37}$ Copyright (2011) John Wiley and Sons

to the temperature in the experiments being beyond $1000 \mathrm{~K}$, favouring considerably thermal cracking reactions.

Concerning applications in presence of oxidants, in addition to the ones mentioned previously, the radicals considered generally include the atomic radical $O$, the hydroxyl radical $O H$ and various de-hydrogenated alcohols, aldehydes and ketones. De Bie et al. showed that $\mathrm{O}, \mathrm{CHO}, \mathrm{OH}$ and $\mathrm{HO}_{2}$ were the most populated radicals (with densities ranging between $10^{9}$ to $10^{14} \mathrm{~cm}^{-3}$ ) when the gas mixture's composition was 70/30 in $C$ $\mathrm{H}_{4} / \mathrm{CO}_{2}$ (see Figure 5). ${ }^{32}$ Nonetheless, the densities of all these radicals varied in function of the discharge features, the methane/oxidant ratio and the nature of the oxidant. Indeed, when the mixture was $\mathrm{CH}_{4} / \mathrm{O}_{2}$ at a 70/30 ratio for the same discharge conditions, the most populated radicals were $\mathrm{CH}_{3} \mathrm{O}_{2}$, $\mathrm{HO}_{2}, \mathrm{O}, \mathrm{CH}_{3} \mathrm{O}$, and $\mathrm{OH}$. All these radicals are commonly

(a) $\mathrm{CH}_{4} / \mathrm{O}_{2}=70 / 30$

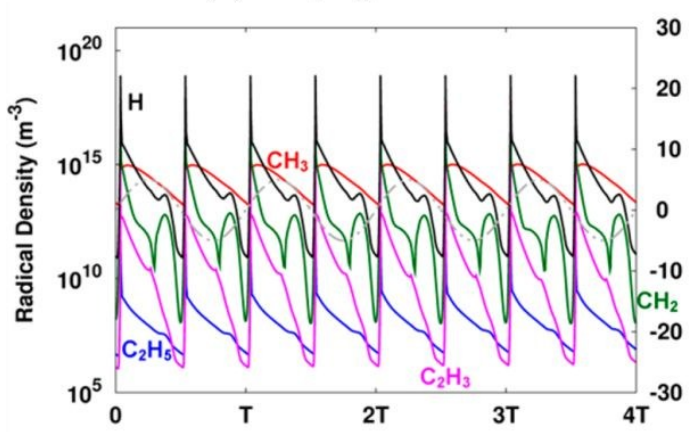

(c) $\mathrm{CH}_{4} / \mathrm{CO}_{2}=70 / 30$

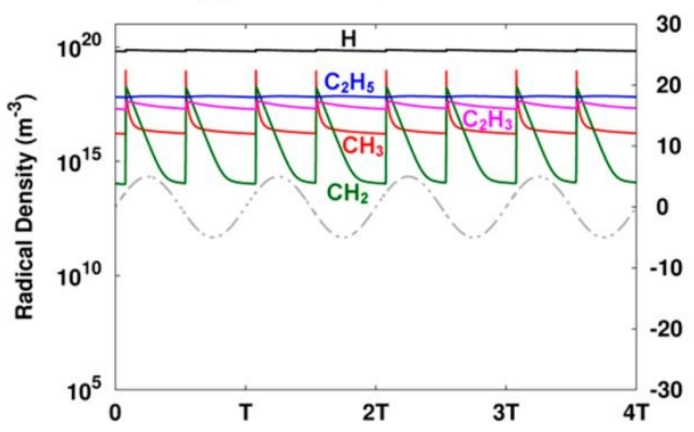

considered in methane combustion studies, wherewthey bave been shown to reach similar number densities.101009/DORE00024H

\subsection{Excited species}

Collisions with electrons can also result in the excitation of the colliding specie. Most of the times, kinetic models only account for the excitation of molecules for the sake of simplicity, however ions and radicals can also become excited ${ }^{101-104}$. The quantitative estimation of the densities of the latter, though, is not an easy task as relevant experimental data are rare.

The excitation mechanisms are governed by quantum mechanics with finite and unvarying energy levels. On a molecular thermodynamics' perspective, an excitation corresponds to an increase of the internal energy of a molecule. There are 3 main ways for a specie to be excited:

- Rotational excitation (low threshold energy, <0.1 $\mathrm{eV} / \mathrm{molecule} \approx 9.65 \mathrm{~kJ} / \mathrm{mol})$

- Vibrational excitation (medium threshold energy between 0.1 to $1.5 \mathrm{eV} /$ molecule)

- Electronical excitation (high threshold energy typically beyond $1.5 \mathrm{eV} / \mathrm{molecule} \approx 145 \mathrm{~kJ} / \mathrm{mol}$ )

Vibrationally excited states of methane have been experimentally observed by Nozaki et al., among others, via optical emission spectroscopy (OES)..$^{43,105-107}$ Such diagnostics can bring valuable insights on the excited species populating the plasma, further allowing the measurement of its vibrational, $T_{v}$, and rotational, $T_{r}$, temperature. Nozaki et al. ${ }^{105}$ estimated the bulk, rotational and vibrational temperatures via OES based on the $C H\left(A^{2} \Delta\right)$ band, while Gao et al. ${ }^{106}$ used the $C_{2}$ Swan band to estimate the vibrational temperature. The vibrational and rotational temperatures in non-thermal plasmas are generally

(b) $\mathrm{CH}_{4} / \mathrm{O}_{2}=70 / 30$

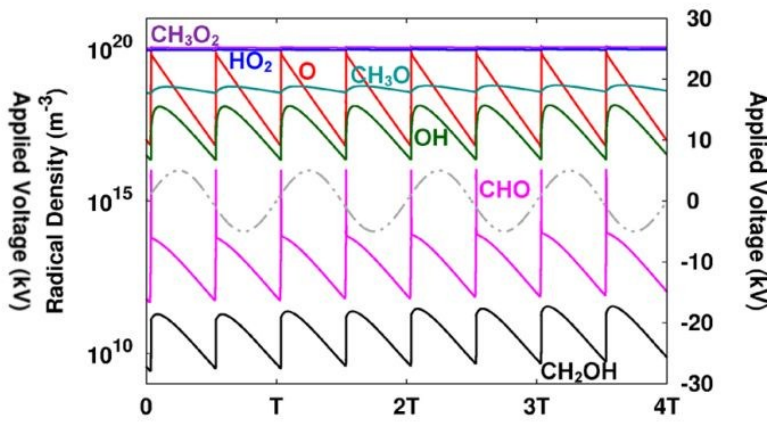

(d) $\mathrm{CH}_{4} / \mathrm{CO}_{2}=70 / 30$

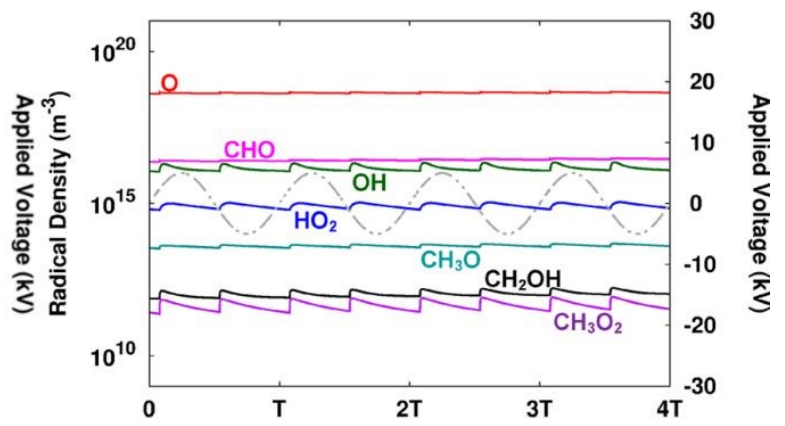

Figure 5. Spatially averaged radical densities for different mix of methane with oxidants. Reprinted with permission from De Bie et al. ${ }^{32}$ Copyright (2015) American Chemical Society 
located between the temperature of the bulk and the electron temperature. From their value, the population of excited states can then be approximated according to the following: ${ }^{108,109}$

$$
T_{v / r}=-\frac{E_{v / r}}{k_{B} \log \left(n_{v / r} / n_{0}\right)}
$$

where $E_{v / r}$ is the energy threshold of the first vibrational/rotational level, $k_{B}$ is the Boltzmann constant, $n_{v / r}$ is the population of excited species and $n_{0}$ the population of the respective ground state.

Inclusion of vibrationally excited hydrocarbons during kinetic modelling has been considered in sources of different backgrounds. Yang accounted for a metastable version of methane $\mathrm{CH}_{4}^{*}$ and 2 excited radicals $\mathrm{H}^{*}$ and $\mathrm{CH}^{*}{ }^{36}$ Later on, Nozaki et al. distinguished two vibrationally excited modes, $\mathrm{CH}_{4}$ (v13) and $\mathrm{CH}_{4}(v 24)$, that were estimated to channel a significant $40 \%$ of the inputted electric energy consumption. ${ }^{105}$ These two modes are actually lumps of close in energy levels $v 1$ and $v 3$ that are stretching modes (symmetric and asymmetric), and $v 2$ and $v 4$ that are bending modes (symmetric and asymmetric) as discussed by Yardley and Moore ${ }^{110}$ and shown in Table 1.

During the same decade, De Bie et al. ${ }^{37}$ and Herrebout et al..$^{53}$ both published models including the same 15 vibrationally excited species: $\mathrm{CH}_{4}(v 13), \mathrm{CH}_{4}(v 24), \mathrm{C}_{2} \mathrm{H}_{6}(v 1), \mathrm{C}_{2} \mathrm{H}_{6}(v 2), \mathrm{C}_{2}$ $\mathrm{H}_{6}(v 3), \mathrm{C}_{2} \mathrm{H}_{4}(v 1), \mathrm{C}_{2} \mathrm{H}_{4}(v 4), \mathrm{C}_{2} \mathrm{H}_{2}(v 13), \mathrm{C}_{2} \mathrm{H}_{2}(v 2), \mathrm{C}_{2} \mathrm{H}_{2}(v$ 5), $C_{3} H_{8}(v 1), C_{3} H_{8}(v 2), H_{2}(v 1), H_{2}(v 2)$, and $H_{2}(v 3)$. Nonetheless, these species were not accounted for separately in the mass balance but were only included in the model for the correct consideration of energy consumption. Recently, Sun and Chen presented a model including the previously mentioned species and adding a vibrationally excited level for propylene $C_{3}$ $H_{6}(v 1) .{ }^{75}$ In this work, the authors accounted for each individual excited state in the mass balance, as a distinct species, so as to study their reactivity. They showed that $\mathrm{CH}_{4} \mathrm{C}$ $v)$ and $H_{2}(v)$ promote chain propagation reactions, which significantly contribute to $\mathrm{C}_{2} \mathrm{H}_{4}$ formation. The method used to estimate the reactions involving vibrationally excited species is further discussed in Section 3.2.3.

Table 1. Vibrational modes of methane

\begin{tabular}{|c|c|c|c|}
\hline Name & Mode & Energy (eV) & Wave number $\left(\mathrm{cm}^{-1}\right)$ \\
\hline$v 1$ & Symmetric stretching & 0.362 & 3147 \\
\hline$v 2$ & Twisting & 0.190 & 1666 \\
\hline$v 3$ & Asymmetric stretching & 0.374 & 3245 \\
\hline$v 4$ & Scissoring & 0.162 & 1453 \\
\hline
\end{tabular}

Accounting for electronically excited states in methane plasma modelling has so far been only superficially explored. The electronically excited modes of methane have indeed been reported to be dissociative $\mathrm{e}^{37,111}$ and have, hence, not been included in most kinetic schemes for reasons of simplicity Nonetheless, this phenomenon is RQt: 1 traej9/fOREORarger hydrocarbons and hydrogen states, meaning that accounting for the respective electronically excited states in plasma kinetic models is a feature currently missing in the literature. Crosssections accounting for the formation of electronically excited states of methane and other relevant hydrocarbons by electron-collision are available on several databases, ${ }^{112-115}$ which means that this improvement on current plasma kinetic schemes should be straightforward. These species contain a significant amount of internal energy, which leads them to ionise or dissociate almost instantaneously. As such, including the reactive processes that involve them could be a determining step in further elucidating methane plasma-chemistry. Moreover, electronically excited states can be responsible for bond-breaking in other molecules leading to the creation of ions or radicals via the so-called Penning ionisation/dissociation phenomenon. ${ }^{116}$ This is verified, in particular, in mixtures with noble gases, where the electronically excited species are populous and can act as energy carriers without leading to the formation of unselective products, such as $\mathrm{CO}_{x}$, as in the presence of oxidants. Experimentally, mixtures of methane and noble gases were thoroughly studied by Jo et al. who investigated the upgrading of methane in the presence of $\mathrm{He}$, $\mathrm{Ne}, \mathrm{Ar}, \mathrm{Kr}$ and $\mathrm{Xe} .{ }^{117-119}$ For the same operating conditions the authors observed a significant increase in methane conversion when the latter was diluted with $K r, X e$, and $A r .119$ This difference with other noble gases was attributed partly to the more pronounced presence of electronically excited atoms, the latter's density being inversely proportional to the excitation potential of their respective ground state (see Table 2 ).

Table 2. Noble gases studied by Jo et al. ${ }^{119}$ Data retrieved with permission from Zhu et al. ${ }^{120}$ Copyright (1990) AIP Publishing

\begin{tabular}{|c|c|c|c|}
\hline Gas & Atomic weight & Excited state & Energy level $(\mathrm{eV})$ \\
\hline$H e$ & 4 & ${ }^{3} S_{1}$ & 19.85 \\
\hline$N e$ & 20 & ${ }^{3} P_{2}$ & 16.64 \\
\hline$A r$ & 40 & ${ }^{3} P_{2}$ & 11.55 \\
\hline$K r$ & 84 & ${ }^{3} P_{2}$ & 9.92 \\
\hline$X e$ & 131 & ${ }^{3} P_{2}$ & 8.32 \\
\hline
\end{tabular}

Concerning the consideration of electronically excited species in kinetic models, the combustion literature provides the most comprehensive sources. Even so, the focus in such works is understandably placed mainly on the excited levels of $N_{2}$ and $\mathrm{O}_{2}$ rather than those of hydrocarbons. Moreover, the vibrationally and rotationally excited species are typically of minor importance in such high temperature systems. A comprehensive example is found in the work of Adamovich et al., who included over 20 excited states, among which multiple vibrational and electronical levels for both $N_{2}$ and $O_{2}$, along with excited metastable atoms $N\left({ }^{2} P^{2}, D\right)$ and $O\left({ }^{1} D,{ }^{1} S\right) .67$

Concerning the reactive low temperature plasmas, the importance of the two metastables $N_{2}\left(A^{3} \Sigma_{u}{ }^{+}\right)$and $N_{2}\left(a^{\prime 1} \Sigma_{u}{ }^{-}\right.$ ) was demonstrated in Snoeck et al. as the species were shown to lead to an increase in the conversion of methane when the 
content in nitrogen was higher than $17.5 \% .{ }^{48}$ These results were attributed to the Penning dissociation previously introduced, according to the following reaction:

$$
\mathrm{N}_{2}\left(A^{3} \Sigma_{u}^{+}\right)+\mathrm{CH}_{4} \rightarrow \mathrm{CH}_{3}+\mathrm{H}+\mathrm{N}_{2}
$$

\section{Plasma-chemical processes}

Following the selection of the species of interest, the processes through which the former interact can be determined and their rates estimated. Numerous elementary reactions are taking place in the plasma-phase simultaneously, each species being involved in multiple formation and consumption processes. These can be divided into two major categories: electron collisions and chemical reactions. The first category corresponds to collisions between an electron and a heavy particle, which can result in several different types of processes as described further below. The second category corresponds to the chemical reactions that occur amongst all the reactive species. The aim of this section is to give an overview of all the processes that are considered in the methane plasma-chemical kinetic models to date and discuss the methods relating to their rates calculation.

\subsection{Electron collisions}

The electron collisions are elementary reactions, whose rate constant $k_{i}$ can be calculated numerically by integration of the collision cross section over the electron energy distribution function (EEDF):

$$
k_{i}=\int_{\varepsilon_{t h}}^{\infty} \sigma_{i}(\varepsilon) v(\varepsilon) f(\varepsilon) d \varepsilon
$$

where:

- $\sigma_{i}(\varepsilon)$ is the cross-section of the considered collision. The cross section is the area within which the two colliding bodies can be assumed to be reacting. In the case of electron collisions, the cross-section is strongly dependent of the electron energy. Except for elastic collisions, all the collision processes have an energy threshold $\varepsilon_{t h}$. This corresponds to an activation energy that must be brought by the electron for the collision to be effective and is removed from the electron upon successful collision.

- $v(\varepsilon)$ is the drift velocity of the electrons that can be estimated upon solution of the Boltzmann equation for the electrons.

- $f(\varepsilon)$ is the EEDF discussed above.

As seen, all these parameters are functions of the electron energy $\varepsilon$ that depends strongly on the shape of the EEDF (see discussion in Section 2.1).

For the case of collisions with a methane molecule, several types of processes can occur, which are described in the following sections. Clearly, within a methane plasma equivalent processes take place also among electrons and the rest of the species besides methane, but these are not explicitly shown below for the sake of brevity. The most comprehensive sources

This journal is (C) The Royal Society of Chemistry 20xx to the authors' opinion to estimate the rates of all described processes are also provided.

DOI: 10.1039/DORE00024H

3.1.1. Elastic collisions. In this type of process, the internal energy of the colliding species does not change, and the total kinetic energy is conserved as well. The fraction of the energy of the electron that is transmitted to the heavy particle is $\gamma=\frac{2 m}{M}$, where $\boldsymbol{m}$ is the mass of the electron and $\boldsymbol{M}$ the mass of the colliding partner. A gain of kinetic energy at the molecular scale corresponds to an increase of the bulk temperature of the gas at macroscale. For methane, the elastic collision can be written as follows, with equivalent processes being defined for other species:

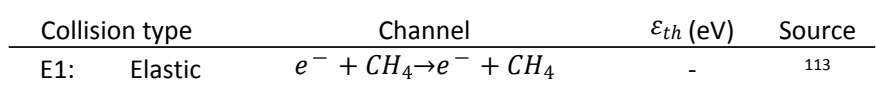

These processes do not affect the mass balance of individual species, but they do have an impact on the energetics of the plasma. The energy fraction that is lost into different channels by electron collisions in a pure methane plasma over a wide range of reduced fields and mean electron energies is shown in Figure 6. When comparing the energy lost in elastic collisions against other channels, it is evident that this process is of minor importance for this type of plasma. Indeed, for reduced fields below approximately $100 \mathrm{Td}\left(1 \mathrm{Td}=1.0 \times 10^{-21} \mathrm{~V} . \mathrm{m}^{2}\right)$, the energy in methane plasmas is mostly lost towards the vibrational excitation channel (see Section 3.1.2). For this reason, momentum transfer reactions are often omitted during methane plasma modelling, or, when included, it is only for the stable molecules. ${ }^{37}$ Elastic collision cross-section data can be found on various databases, ${ }^{112-115}$ however it is important to note that the often called "effective" or "momentum" crosssections are total cross-sections encompassing both elastic and inelastic momentum transfer processes. It is, thus, important when building kinetic schemes that, if available, real elastic cross-sections are used.

3.1.2. Excitation. These processes describe collisions where the electron transmits part of its energy to a ground-state molecule,

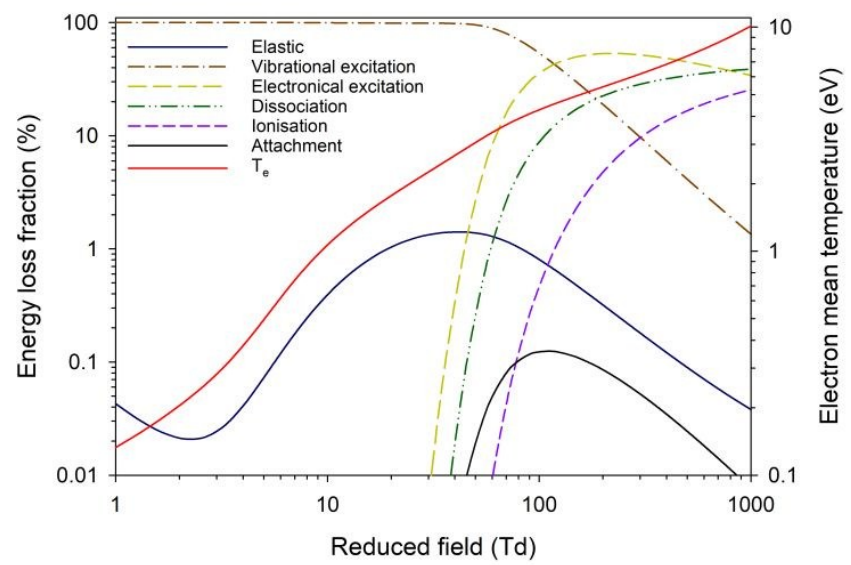

Figure 6. Energy channelling in a pure methane plasma at atmospheric pressure. Results obtained with BOLSIG+. Cross section data derived from LXCat. ${ }^{113,114,121}$ The average electron temperature $T_{e}$ is obtained from the EEDF. 
resulting in the excitation of the latter. The threshold energy of the reaction is transferred to the internal energy of the species. Depending on the mode of excitation (see Section 2.5) this internal energy varies. As discussed above, the vibrational excitation of methane by electron collisions is the dominant energy loss channel when the reduced field is below $100 \mathrm{Td}$ in a pure methane plasma (see Figure 6). When the field is beyond $100 \mathrm{Td}$ the electronical excitation of methane becomes dominant instead. These processes for methane are summarised below, noting again that modes $v 24$ and $v 13$ are lumps of modes $v 2$ and $v 4$, and $v 1$ and $v 3$, respectively, considered so in most published modelling work due to the proximity of the energy thresholds of the individual modes within each lump:

\begin{tabular}{ccccc}
\multicolumn{2}{c}{ Excitation type } & \multicolumn{1}{c}{ Channel } & $\varepsilon_{\text {th }}(\mathrm{eV})$ & Source \\
\hline EV1: & Vibrational & $e^{-}+\mathrm{CH}_{4} \rightarrow e^{-}+\mathrm{CH}_{4}(v 24)$ & 0.162 & 114 \\
EV2: & Vibrational & $e^{-}+\mathrm{CH}_{4} \rightarrow e^{-}+\mathrm{CH}_{4}(v 13)$ & 0.361 & ${ }_{114}$ \\
EE1: & Electronical & $e^{-}+\mathrm{CH}_{4} \rightarrow e^{-}+\mathrm{CH}_{4} *(7.9 \mathrm{eV})$ & 7.9 & ${ }_{113}$
\end{tabular}

Different approaches have been applied to account for excitation in methane plasma kinetic modelling literature. As the implementation of such processes leads to the creation of new species, increasing the complexity of the models significantly, several studies have included these product species only as energy sinks for the purpose of the proper determination of the EEDF. ${ }^{29,32,37}$ To account for the impact of the energy losses associated with excitation, Snoeckx et al. considered several cases of what the authors named "plasma power transfer efficiency" where only $75 \%$ or $50 \%$ of the plasma power is transferred efficiently to the chemical reaction processes. $^{29}$ This was performed in order to compare the simulation results with experimental data, finding that the closest agreement in energy efficiency was achieved under the most restrictive scenario of 50\% energy being lost on excitation. It is indeed important to consider excitation processes to correctly evaluate the efficiency of the energy channelling in the plasma process. Along this line, as discussed in Section 2.5, it was estimated by Nozaki et al. that the energy lost towards methane excitation in pure methane plasma was reaching up to $40 \%$ of the total energy. ${ }^{43,105}$ Sun and Chen estimated the energy loss fraction in methane excitation to be varying between $8 \%$ to $37 \%$ in a helium diluted methane plasma for reduced electric field values between 40 and $90 \mathrm{Td} .{ }^{75}$

3.1.3. Ionisation. These are essential processes of the plasma phase as they are the primary sources of electrons. In nonthermal plasmas the direct ionisation by electron collision is the dominant pathway. ${ }^{88}$ The dissociative ionisation is distinguished by the fact that the electron collisions result in the production of an ion and other neutral species. The threshold energies of dissociative ionisations are always higher than those of nondissociative ionisations, with all being generally over $10 \mathrm{eV}$ :
Ionisation type

Channel

Etale (eVt)iclesourige

11: Non-dissociative $e^{-}+\mathrm{CH}_{4} \rightarrow 2 e^{-}+\mathrm{CH}_{4}^{+}$DOI: 10.1039/R2.6E00024H

$\begin{array}{lllll}\text { 12: } & \text { Dissociative } & e^{-}+\mathrm{CH}_{4} \rightarrow 2 e^{-}+\mathrm{CH}_{3}^{+}+\mathrm{H} & 14.3 & 121 \\ \text { 13: } & \text { Dissociative } & e^{-}+\mathrm{CH}_{4} \rightarrow 2 e^{-}+\mathrm{CH}_{2}^{+}+\mathrm{H}_{2} & 15.1 & 121 \\ \text { 14: } & \text { Dissociative } & e^{-}+\mathrm{CH}_{4} \rightarrow 2 e^{-}+\mathrm{CH}^{+}+\mathrm{H}_{2}+\mathrm{H} & 19.9 & 121\end{array}$

The onset of ionisation occurs at fields of about $80 \mathrm{Td}$ in pure methane (see Figure 6), however the process remains minor in the non-thermal plasma domain in terms of energy consumption ( $\approx 1-2 \%$ of the total energy consumption). Most of the global ionisation products are $\mathrm{CH}_{4}^{+}$, accounting for $80 \%$ of the overall ionisation.

Depending on the application, the level of detail applied to ionisation processes during modelling has varied. The most comprehensive sources originate from the PLASMANT group and relate to the non-oxidative, ${ }^{37}$ oxidative, ${ }^{32}$ and in the presence of $\mathrm{N}_{2}{ }^{48}$ upgrading of methane in a Dielectric Barrier Discharge. In these models, ionisation reactions involving all individual neutral species (molecules and radicals) are included explicitly. It is found that the first ionisation (I1) and the first dissociative ionisation (12) of methane are the main sources of electrons, accounting for 66 and $33 \%$ of total electron production, respectively. 32,37 When mixed with nitrogen, ionisations 11 and 12 still remain the main sources of electrons up to a $88 \% N_{2}$ content due to their lower energy thresholds in comparison to the first ionisation of nitrogen $(15.6 \mathrm{eV}) .^{48}$

3.1.4. Dissociation. In such processes, the collision between the electron and the heavy particle results in bond-breaking and the production of smaller radicals. These reactions are the main sources of radicals in non-thermal plasmas. The threshold energies are in the range of 5-10 eV and vary depending on the molecule:

\begin{tabular}{clcc} 
& \multicolumn{1}{c}{ Channel } & $\varepsilon_{\text {th }}(\mathrm{eV})$ & Source \\
\hline D1: & $e^{-}+\mathrm{CH}_{4} \rightarrow e^{-}+\mathrm{CH}_{3}+\mathrm{H}$ & 8.8 & 121 \\
D2: & $e^{-}+\mathrm{CH}_{4} \rightarrow e^{-}+\mathrm{CH}_{2}+\mathrm{H}_{2}$ & 9.4 & 121 \\
D3: & $e^{-}+\mathrm{CH}_{4} \rightarrow e^{-}+\mathrm{CH}+\mathrm{H}+\mathrm{H}_{2}$ & 12.5 & 121 \\
D4: & $e^{-}+\mathrm{CH}_{4} \rightarrow e^{-}+\mathrm{C}+2 \mathrm{H}_{2}$ & 14.0 & 121
\end{tabular}

The most comprehensive kinetic schemes are the same as those previously mentioned for ionisation, ${ }^{32,37,48}$ implementing the different steps of dehydrogenation and fragmentation for each individual hydrocarbon in an elaborate way. Dissociation D1 has been identified as the initial methane consuming process in numerous modelling papers. ${ }^{17,29,32,36,37,43,48,75}$ This is a direct consequence of its lower energy threshold comparatively with the other electron-dissociations. Nonetheless, the relative importance of this process on the total methane conversion varies significantly among reported values. For a pure methane plasma, De Bie et al. ${ }^{37}$ found the dissociation D1 to account for approximately $38 \%$ of total methane consumption, while Yang ${ }^{36}$ estimated this to be about $18 \%$. This discrepancy could be due to a variety of reasons (different discharge features, different electron energy distribution function, different kinetic 
parameters, etc.), making, as such, a quantitative comparison between such works hard to perform.

3.1.5. Attachment reactions. These processes are the sources of negative ions when included. Like ionisation, attachment reactions can also lead to the dissociation of the molecule. The threshold energies of such processes are very close to zero for electro-negative species, however for methane, being an electropositive species, this is not the case. Davies et al. identified two dissociative attachment reactions producing $\mathrm{H}^{-}$ and $\mathrm{CH}_{2}^{-}$with threshold energy of $7.7 \mathrm{eV}$ and $9 \mathrm{eV}$, respectively: ${ }^{96}$

\begin{tabular}{ccccc}
\multicolumn{2}{c}{ Attachment type } & Channel & $\varepsilon_{t h}(\mathrm{eV})$ & Source \\
\hline DA1: & Dissociative & $e^{-}+\mathrm{CH}_{4} \rightarrow \mathrm{H}_{2}+\mathrm{CH}_{2}^{-}$ & 7.7 & 96 \\
DA2: & Dissociative & $e^{-}+\mathrm{CH}_{4} \rightarrow \mathrm{CH}_{3}+H^{-}$ & 9.0 & 96
\end{tabular}

As confirmed from Figure 6, these reactions are of small importance to methane plasma modelling as they account for only $0.1 \%$ of the overall energy lost by the electrons. For this reason, most of the relevant studies have indeed omitted these reactions for the hydrocarbons. In cases involving oxygenated species attachment reactions have to be included as they represent a significant channel of creation of negative ions, the latter affecting the discharge pattern ${ }^{32}$ and also constituting a non-negligible contribution to the material balance. Indicatively, the dissociative attachment of an electron to $\mathrm{CO}_{2}$, according to $e^{-}+\mathrm{CO}_{2} \rightarrow \mathrm{O}^{-}+\mathrm{CO}$, was found to account for approximately $3 \%$ of total $\mathrm{CO}_{2}$ consumption in a $70 / 30 \mathrm{CH}_{4} / \mathrm{C}$ $\mathrm{O}_{2}$ mixture. $^{32}$

3.1.6. Dissociative recombination. These processes describe the collision between a positive ion and an electron, which results in the production of 2 neutral particles. These reactions proceed via an excited state that dissociates because of the energy that was brought by the electron:

$$
e^{-}+A B^{+} \rightarrow(A B)^{*} \rightarrow A+B^{*}
$$

The recombination mechanism is fast and is very important in molecular plasmas, but it can also take place in atomic plasmas in a process involving an additional electron as a third body partner:

$$
\begin{gathered}
e^{-}+e^{-}+A^{+} \rightarrow e^{-}+A^{*} \\
A^{*} \rightarrow A+h \omega
\end{gathered}
$$

As seen above, the excited intermediate atom $A^{*}$ further on loses the recombination energy by emitting a photon. Other heavy particles can't act as the third body partner as they are unable to accommodate the recombination energy of the electron fast enough in their kinetic energy. ${ }^{88}$

The rate coefficients of these reactions decrease with the increase of the electron temperature and have no energy threshold. They are generally described with several possible branching channels when the heavy particle is a molecule. ${ }^{88}$ Janev and Reiter proposed several correlations in function of the electron temperature to estimate the rate constant for nall hydrocarbon ions pertinent to methaRel:pRaspha/DPBodening, including all the possible branching channels with their respective branching ratios. ${ }^{121,122}$ For the methane ion these are summarised below:

\begin{tabular}{clcc} 
& \multicolumn{1}{c}{ Channel } & Branching ratio & Source \\
\hline DR1: & $e^{-}+\mathrm{CH}_{4}^{+} \rightarrow \mathrm{CH}_{3}+\mathrm{H}$ & 0.21 & 121 \\
DR2: & $e^{-}+\mathrm{CH}_{4}^{+} \rightarrow \mathrm{CH}_{2}+\mathrm{H}_{2}$ & 0.09 & 121 \\
DR3: & $e^{-}+\mathrm{CH}_{4}^{+} \rightarrow \mathrm{CH}_{2}+\mathrm{H}+\mathrm{H}$ & 0.43 & 121 \\
DR4: & $e^{-}+\mathrm{CH}_{4}^{+} \rightarrow \mathrm{CH}+\mathrm{H}_{2}+\mathrm{H}$ & 0.25 & 121 \\
DR5: & $e^{-}+\mathrm{CH}_{4}^{+} \rightarrow \mathrm{C}+2 \mathrm{H}_{2}$ & 0.02 & 121
\end{tabular}

Due to the low densities of both ions and electrons, the production of radicals through this route is several orders of magnitude lower than that from other processes (direct dissociation of hydrocarbons, radical-radical reactions, etc.). Nonetheless, it is still important to include these reactions in kinetic schemes as they are the dominant mechanisms through which electrons and positively charged species can be consumed in the plasma phase. The most elaborate consideration of DR processes for methane plasmas, with several channels for individual ions systematically included, was realised in the works of the PLASMANT group. ${ }^{29,32,37,48}$ Other than that, a certain degree of detail for hydrocarbon ions and also $\mathrm{O}_{2}$ or $\mathrm{N}_{2}$ can also be found in some plasma assisted combustion studies. $68,69,80$

\subsection{Chemical processes.}

Following the formation of the primary reactive species via electron collisions, further reactions amongst these species can occur. The numerical simplicity of kinetic modelling allows for a high level of detail in the amount of processes that are included. Therefore, the number of considered reactions in methane kinetic schemes presented in literature has consistently grown. One of the earliest non-oxidative methane conversion kinetic schemes considered 56 chemical reactions, 23 being neutralneutral and 33 ion-neutral steps, ${ }^{52}$ whereas more recently the most comprehensive scheme accounted for 948 reactions, ${ }^{75}$ indicative of the rapid expansion of such networks.

3.2.1. Neutral-neutral reactions. This category incorporates radical chemistry pathways, such as chain propagation and coupling reactions, that lead to final products and further radical formation. Typical processes from methane thermal decomposition are included that overall follow the phenomenological Kassel mechanism. ${ }^{123}$ These types of reactions have been extensively studied in the frame of methane combustion and pyrolysis, with various sources compiling elaborate reaction schemes and rate constants. ${ }^{124-128}$ The latter are typically a function of temperature under the formalism of an Arrhenius-type law. The considerable amount of possible reactions does not allow to explicitly comment on all. Nonetheless, it needs to be noted that the rates of these reactions expand across several orders of magnitude, with only 
a subset of reactions being typically responsible for the net conversion. Comparing a variety of sources, ${ }^{36-38,75}$ the most important neutral-neutral reactions in non-oxidative methane conversion are the following:

$\begin{array}{lc}\text { NN1: } & \mathrm{CH}_{3}+\mathrm{CH}_{3}+\mathrm{M} \rightarrow \mathrm{C}_{2} \mathrm{H}_{6}+\mathrm{M} \\ \text { NN2: } & \mathrm{CH}_{2}+\mathrm{CH}_{2} \rightarrow \mathrm{C}_{2} \mathrm{H}_{2}+\mathrm{H}_{2} \\ \text { NN3: } & \mathrm{H}_{2}+\mathrm{CH}_{2} \rightarrow \mathrm{CH}_{3}+\mathrm{H} \\ \text { NN4: } & \mathrm{C}_{2} \mathrm{H}_{5}+\mathrm{C}_{2} \mathrm{H}_{5} \rightarrow \mathrm{C}_{2} \mathrm{H}_{6}+\mathrm{C}_{2} \mathrm{H}_{4} \\ \text { NN5: } & \mathrm{C}_{2} \mathrm{H}_{5}+\mathrm{H} \rightarrow \mathrm{CH}_{3}+\mathrm{CH}_{3} \\ \text { NN6: } & \mathrm{C}_{2} \mathrm{H}_{4}+\mathrm{H}+\mathrm{M} \rightarrow \mathrm{C}_{2} \mathrm{H}_{5}+\mathrm{M} \\ \text { NN7: } & \mathrm{C}_{2} \mathrm{H}_{5}+\mathrm{H}+\mathrm{M} \rightarrow \mathrm{C}_{2} \mathrm{H}_{6}+\mathrm{M}\end{array}$

As discussed previously, the production of $\mathrm{CH}_{3}$ and $\mathrm{CH}_{2}$ radicals originates mainly from the dissociation of methane via electron collisions. Once formed, these radicals can further couple and lead to the production of $\mathrm{C}_{2} \mathrm{H}_{6}$ and $\mathrm{C}_{2} \mathrm{H}_{2}$ (reactions NN1 and NN2). Ethane, a primary product of the process, can further dehydrogenate via electron collisions towards the secondary product $\mathrm{C}_{2} \mathrm{H}_{4}$, which can also dehydrogenate via similar processes (this corresponds to the aforementioned Kassel mechanism). In parallel, it is also possible for $\mathrm{C}_{2} \mathrm{H}_{4}$ to hydrogenate back up to $\mathrm{C}_{2} \mathrm{H}_{6}$ according to reactions NN6 and NN7. The main features of such contribution analyses are shown in Figure 7 as obtained from two different literature sources.

It is important to note here that many of the neutral-neutral reactions (for example NN1, NN6 and NN7 above) are threebody processes. In this type of reaction two species $A$ and $B$ yield a single excited specie $A B^{*}$. A third body $M$ is required to stabilise $A B^{*}$, which would otherwise self-dissociate. $M$ eventually releases the excess energy to the gas bulk as heat. The requirement for the presence of $M$, typically assumed to be any available species in the gas, leads to the rate of such reactions being pressure-dependent. The respective rate constants are generally given in literature at two limits: the lowpressure limit $\left(k_{0}\right)$, where the rate is first order in the concentration of $M$, and the high-pressure limit $\left(k_{\infty}\right)$, where it is zero order in the concentration of $M$. A formalism such as that of Lindemann ${ }^{129}$ or Troe ${ }^{130}$ describes the rate constant at intermediate pressures. The chemical nature of the third bady $M$ can also affect the efficiency of the corisibnt, aecolinteded for via efficiency factors.

Certain studies on plasma-assisted combustion and reactive plasmas have treated three-body reactions thoroughly. De Bie et al. ${ }^{32,37}$ accounted for these reactions explicitly, although the third body $M$ density was considered to be equal to that of the most populated molecules while reactions were considered to be either at the low pressure or high pressure limit. Three-body reactions were revealed to be determining for the recombination of methyl radicals (into ethane, propane and methane) acting as the main sinks of $\mathrm{CH}_{3}$. Ravasio and Cavallotti ${ }^{38}$ further considered the pressure dependence using the Troe formalism and observed similar strong effects on the recombination of radicals.

3.2.2. Ion-Neutral reactions. These reactions are important to describe the interaction of ions with the rest of the gas species but are generally of lower significance to the global chemistry. They can be simple charge-exchange reactions:

$$
\mathrm{CH}_{3}^{+}+\mathrm{CH}_{4} \rightarrow \mathrm{CH}_{4}^{+}+\mathrm{CH}_{3}
$$

Alternatively, they can lead to bond breaking such as:

$$
\mathrm{CH}_{2}^{+}+\mathrm{CH}_{4} \rightarrow \mathrm{CH}_{3}^{+}+\mathrm{CH}_{3}
$$

The rate constants of these reactions do not follow an Arrhenius law and are typically assumed in literature to be constants. An experimental evaluation of the reaction of $\mathrm{C}^{+}, \mathrm{CH}^{+}, \mathrm{CH}_{2}^{+}, \mathrm{C}$ $\mathrm{H}_{3}^{+}$, and $\mathrm{CH}_{4}^{+}$with various molecules at $300 \mathrm{~K}$ was presented by Smith and Adams. ${ }^{131}$ The most comprehensive kinetic schemes for such reactions can again be found in the works of the PLASMANT group. ${ }^{32,37,48}$ In these studies, reaction rate constants were obtained from sources, which although comprehensive, rely on theoretical astrochemistry models that refer to much different conditions than those of interest to nonthermal plasma applications. ${ }^{132,133}$

3.2.3. Reactivity of excited states. In recent literature, excited species have been considered to explain the observed rates in
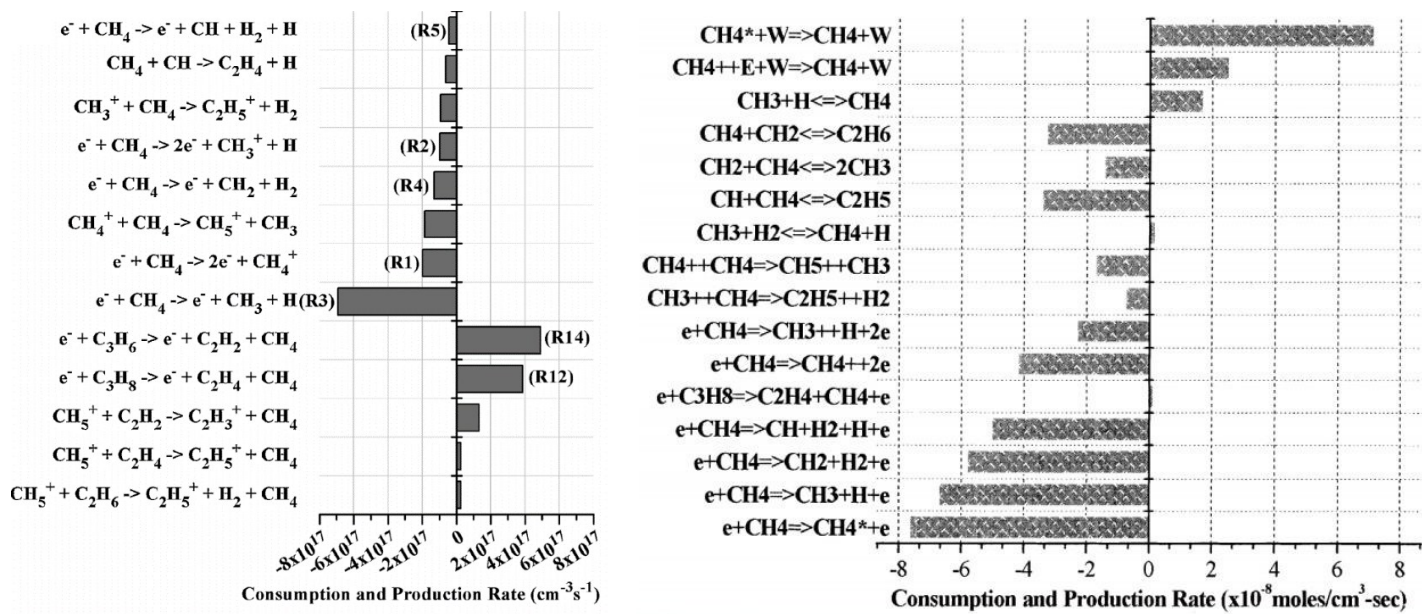

Figure 7. Main consumption and production processes in non-oxidative methane plasma data. Left panel adapted with permission from De Bie et al. ${ }^{37}$ Copyright (2011) American Chemical Society. Right panel reprinted with permission from Yang et al. ${ }^{36}$ Copyright (2003) Springer Nature 
plasma chemical processes. ${ }^{47,48,75}$ As already discussed, accounting for excited states as separate species in kinetic models increases complexity significantly, while the relative lack of experimental data relating to their reactivity further hampers their explicit consideration. This holds also for methane plasma literature, necessitating the use of theoretical approaches for the estimation of relevant reaction rates.

A common assumption made is that excited states react according to the same channels of the ground state, with their higher internal energy contributing in overcoming the activation energy of these reactions. ${ }^{88}$ Effectively this means that reactions involving exited states have the same stoichiometry as those involving the ground-state but proceed at a higher rate. The Fridman-Macheret $\alpha$-Model permits the estimation of the rate coefficient of a reaction that involves a vibrationally excited state: ${ }^{88}$

$$
k_{R}\left(E_{v}, T_{0}\right)=k_{R 0} \exp \left(-\frac{E_{a}-\alpha E_{v}}{T_{0}}\right) \theta\left(E_{a}-\alpha E_{v}\right)
$$

In this formula, $k_{R 0}$ and $E_{a}$ are the pre-exponential factor and activation energy of the reaction rate of the ground-state equivalent reaction, $E_{v}$ is the threshold energy of the vibrationally excited state, $\alpha$ is the efficiency of the excited state to overcome the energy barrier $E_{a}$, and $\theta\left(E_{a}-\alpha E_{v}\right)$ is the Heaviside function according to which

- $\theta\left(E_{a}-\alpha E_{v}\right)=1$ when $\left(E_{a}-\alpha E_{v}\right)>0$

- $\theta\left(E_{a}-\alpha E_{v}\right)=0$ when $\left(E_{a}-\alpha E_{v}\right)<0$

The rate of such a reaction follows the same Arrhenius law as the ground state but with an activation energy that is lowered

a

(a)
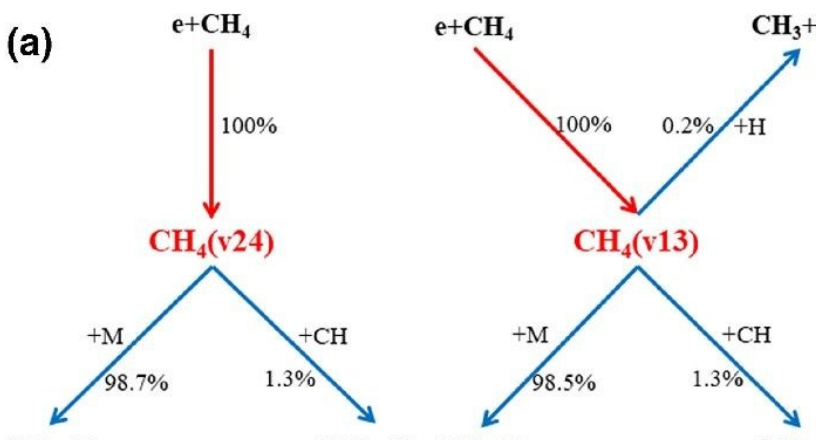

$\mathrm{CH}_{4}+\mathrm{M}$
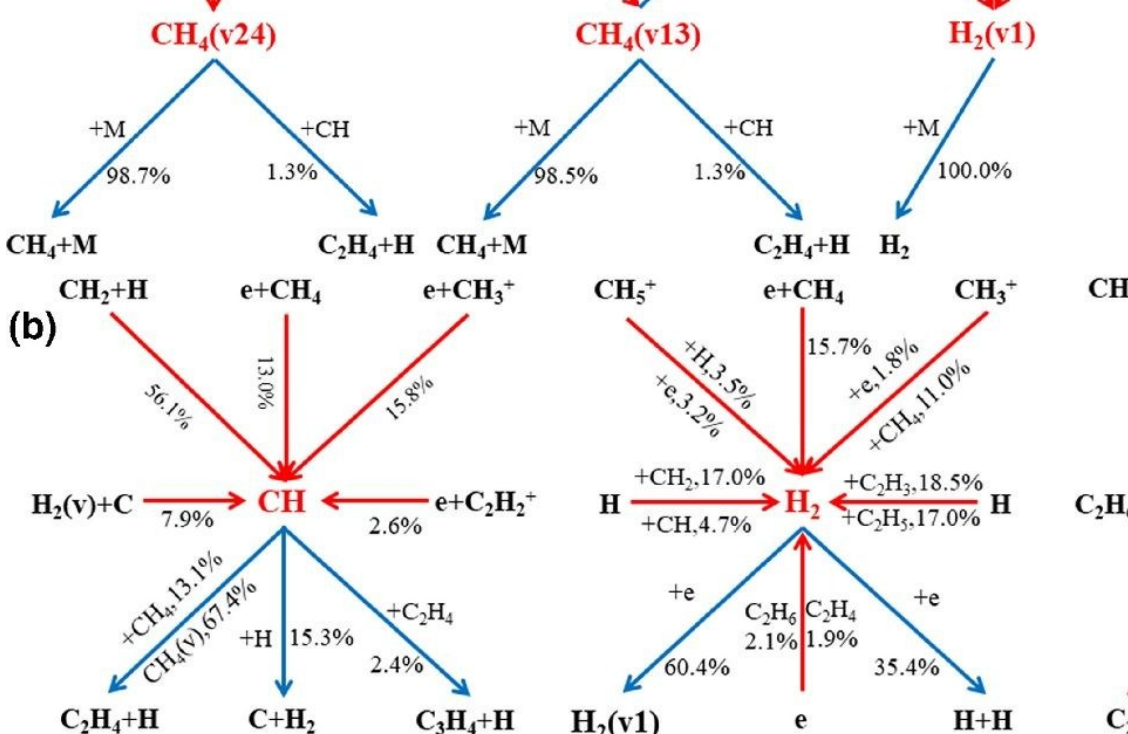

by the vibrational energy and the efficiency factor the tatter determined by the nature of the reaction? If thelpeactionf pesults in bond breaking in both molecules, $\alpha$ ranges between $0.5-0.9$ for an endothermic reaction, $0.1-0.3$ for an exothermic reaction, and $0.3-0.5$ for a thermo-neutral reaction.

To date, the Fridman-Macheret model has been applied in methane plasma modelling only in the work of Sun and Chen. ${ }^{75}$ The authors found that the excited modes of hydrogen $\mathrm{H}_{2}(\mathrm{v} 2)$ and $H_{2}(v 3)$ are quickly consumed by chain propagation reaction $\mathrm{H}_{2}(v)+\mathrm{C} \rightarrow \mathrm{CH}+\mathrm{H}$. The high vibrational energy of these states (1.0 and $1.5 \mathrm{eV}$ for $H_{2}(v 2)$ and $H_{2}(v 3)$, respectively) resulted in their net rate of consumption being 5 orders of magnitude higher than that of ground-state $\mathrm{H}_{2}$. In the same work, vibrationally excited methane $\mathrm{CH}_{4}(v)$ was estimated to account for $31.4 \%$ of $\mathrm{C}_{2} \mathrm{H}_{4}$ production (see reaction pathway analysis in Figure 8 ).

3.2.4. Energy relaxation processes. Important phenomena to account for when considering excited states is the energy relaxation processes, where the internal energy of these states is lost through non-reactive processes. The transfer of energy can take place from vibrational to translation (VT relaxation) energy transitions: ${ }^{88}$

$$
\mathrm{CH}_{4}(v)+M \rightarrow \mathrm{CH}_{4}+\mathrm{M}+\mathrm{E}_{v}
$$

In this case, $\mathrm{CH}_{4}(v)$ returns to the ground-state, losing its quantum of energy in translational degrees of freedom, a process that corresponds on the macroscale to heat production. The collisional energy transfer in gases and the relaxation of excited states has been a matter of study in physics since the rise of quantum mechanics. The Landau-Teller model was the first to be developed in $1936,{ }^{134}$ forming the basis for the SSH

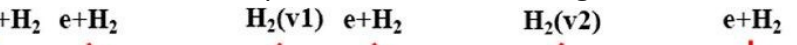
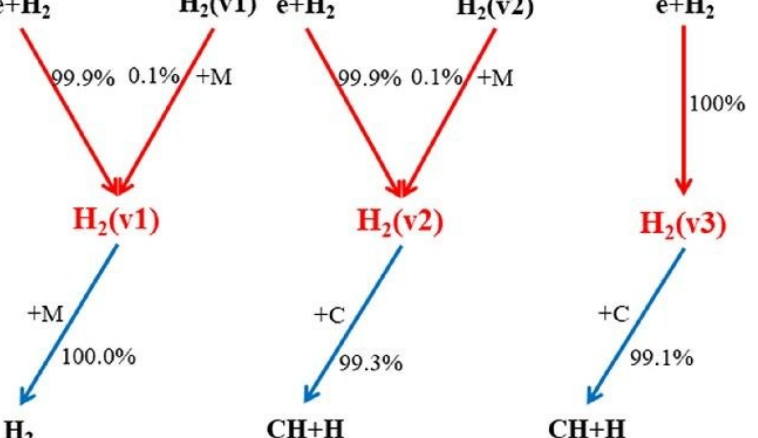

$\mathbf{C H}+\mathbf{H}$

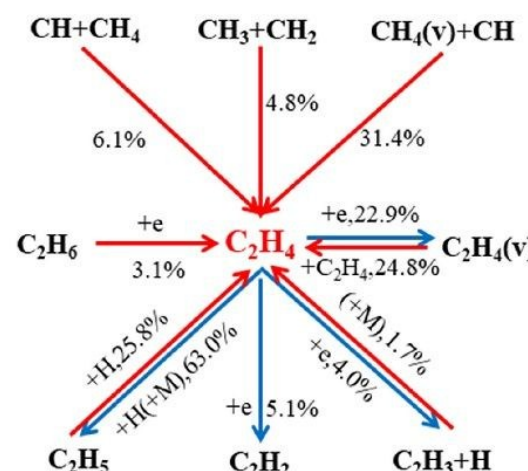

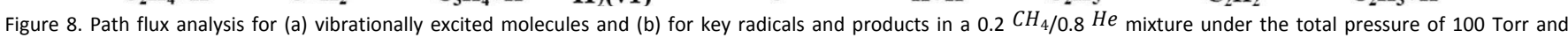
temperature of $373 \mathrm{~K}$, with reduced field of $60.0 \mathrm{Td}$. Reprinted with permission from Sun and Chen. ${ }^{75}$ Copyright (2019) Elsevier 
theory ${ }^{135}$ that became the foundation in explaining vibrational non-equilibrium phenomena. It was subsequently improved into the "infinite order sudden" (IOS), "coupled states" (CS) and "close coupling" (CC) models that are nowadays standard for studying molecular collisions. ${ }^{136}$ Nonetheless, these are not commonly encountered in methane kinetic modelling studies. In 1974, Lifshitz ${ }^{137}$ proposed a semi-empirical relation that allows to estimate the VT rate in function of the colliding partner $M$, which is very convenient when it comes to estimating the rate of de-excitation for each individual process. The relaxation of excited states of methane via VT transfer is to date poorly investigated due to lack to experimental data. Sun and $\mathrm{Chen}^{75}$ accounted for the VT transfer, inferring the rates from Starikovskiy et al. ${ }^{138}$ and finding that $98.7 \%$ of $\mathrm{CH}_{4}(v 24)$ and $98.5 \%$ of $\mathrm{CH}_{4}(v 13)$ were quenched by these processes at the simulated conditions.

Alternatively, vibrational relaxation can take place via vibrational to vibrational (VV) processes, which occur following the collision of two same chemical species at different vibrational excitation levels:

$$
\mathrm{CH}_{4}(v 3)+\mathrm{CH}_{4} \rightarrow \mathrm{CH}_{4}(\mathrm{v} 4)+\mathrm{CH}_{4}(v 4)+\left|E_{v 3}-2 E_{v 4}\right|
$$

The higher vibrational level $v 3$ decays to $v 4$, whereas the ground-state methane is excited to $v 4$. A small amount of energy $\left|E_{v 3}-2 E_{v 4}\right|$ is also released in translation. These types of relaxation processes are much faster than the VT processes and result in a net deexcitation of higher vibrational states to lower ones. There is for now no account of such process in the methane plasma literature. Nonetheless, Yardley et al. investigated the relaxation processes of methane, excited by a $\mathrm{He}-\mathrm{Ne}$ laser, proposing several deexcitation schemes involving VV and VT transfers to model the de-excitation of methane. ${ }^{110}$ This work was performed using the previously mentioned SSH theory, fitted to the authors' experimental data.

\section{Modelling of methane plasmas}

The modelling of methane plasmas has been the subject of numerous investigations. In this section, the various approaches followed to model the plasma are reviewed, while recent findings reported in the relevant literature on the modelling are also discussed.

\subsection{Zero-dimensional kinetic modelling}

The most common approach to modelling a methane plasma is to use a 'zero-dimensional' kinetic code. These codes solve simultaneously a system of conservation equations for the species densities based on calculated reaction rates, and also sometimes the Boltzmann equation, under the a priori assumption of a spatially homogenous discharge. A range of additional terms may be included in the equations of conservation of the species densities to account for additional physics. For example, losses of species to reactor walls, and flows into and out of a reactor. ${ }^{139}$ The following is an example of a set of equations of a OD model:

$$
\begin{aligned}
& \frac{d n_{i}}{d t}=R_{i}\left(\frac{E}{N}, T_{g}\right)-\frac{A}{V} v_{i} n_{i} \frac{n_{i}}{\text { DO }} \begin{array}{r}
\text { View Article Online } \\
\frac{d n_{n}}{d t}=R_{n}\left(\frac{E}{N}, T_{g}\right)-\frac{A}{V} v_{n} n_{n}-\frac{n_{n}}{\tau}+\frac{n_{f}}{\tau}
\end{array}
\end{aligned}
$$

with the electron density, reduced field $(E / N)$, and gas temperature being used as model inputs. The subscripts $i, n, g$, and $f$ refer to ions, neutrally charged species, gas temperature, and the feed gas species, respectively; $n$ is the species number density; $R$ is the reaction rate; $A$ is the surface area of the vessel walls; $V$ is the volume of the reactor vessel; $v$ is the species velocity incident on the vessel walls; $\tau$ is the residence time of the reactor.

Zero-dimensional models have been used to simulate the complex methane plasma reaction networks for a variety of discharge types, including dielectric barrier discharges, ${ }^{36}$ microwave discharges, ${ }^{140}$ radio frequency discharges, ${ }^{52}$ arc discharges, ${ }^{40}$ and also the gas phase kinetics at temperatures reached by arc discharges. ${ }^{141}$ As mentioned above, an advantage of using such codes is that they can simulate very large reaction networks at acceptable computational cost. Also, modelling can be performed for the duration of the reactor residence time, which can be 10 orders of magnitude greater than the characteristic times of electron dynamics. However, these zero-dimensional codes evidently lack the capability of describing the spatial variation of a discharge. Therefore, plasma phenomena like space-charge sheaths ${ }^{45}$, streamer heads, and the filamentary nature of some dielectric barrier discharges, are not possible to be taken into account. The validity of the conclusions based on zero-dimensional modelling of a reactor are, generally, restricted to findings of qualitative trends; except potentially in the case of a spatially homogenous plasma bulk, as in some RF discharges and the column of large DC glows. A more detailed explanation of zero-dimensional codes can be found in the review by Hurlbatt et al. ${ }^{139}$ The following paragraph discusses recent findings from zerodimensional modelling of methane plasma reported in the literature.

In 2003, Yang published a report of a OD modelling study of direct non oxidative conversion of methane gas into higher hydrocarbons. ${ }^{36}$ The residence time of the reactor simulations was reduced to account for the non-homogeneous, filamentary, nature of a DBD discharge and obtain a better description of experimental results. A key finding of the study was that with greater specific energy input, a transition in selectivity from mainly $\mathrm{C}_{2} \mathrm{H}_{6}$ and $\mathrm{C}_{3} \mathrm{H}_{8}$ towards $\mathrm{C}_{2} \mathrm{H}_{4}$, and $\mathrm{C}_{2} \mathrm{H}_{2}$ is observed. In 2005, Indarto and co-authors reported simulation and experimental results of methane conversion by a gliding arc discharge. ${ }^{40} \mathrm{~A}$ kinetic model that considered only gas phase reactions among stable molecules and radicals was implemented. The discharge's high plasma density resulted in good conversion ( $50 \%$ ) for even high methane feed gas flow rates, with a high selectivity towards acetylene. The modelling showed that in the nonequilibrium zone the gas temperature is increased by $\sim 50^{\circ} \mathrm{C}$. In 2006, Benilov and Naidis reported on modelling of the gas phase of an arc discharge in methane using standard literature gas phase kinetic schemes of methane 
oxidation. ${ }^{141}$ The high temperatures generated by the arc were found to be sufficient to convert the methane at similar levels seen in the experiments, without the need for any specific plasma related mechanism to be accounted for. In 2007, Pintassilgo and co-authors reported on modelling of a small quantity of methane passing through the afterglow of a nitrogen microwave plasma, using OD kinetic codes. ${ }^{140}$ The electronically excited nitrogen metastable, $N_{2}\left(A^{3} \Sigma_{u}{ }^{+}\right)$, in the afterglow was shown to collide with methane, deexcite, and dissociate the methane to $\mathrm{CH}_{x}$ radicals. These products then chemically reacted with the nitrogen to form hydrogen cyanide, other $C N$ species and finally $C$ atoms. In 2008, Indarto and coauthors published results of a OD modelling study of direct non oxidative methane conversion by DBD. ${ }^{41}$ Simple first order reactions among stable molecules were considered, while rate coefficients were fitted to experimental data of the work. Nonetheless, in agreement with more complex models the product selectivities were found to be sensitive to the rate of dehydrogenation of $\mathrm{C}_{2} \mathrm{H}_{6}$ into $\mathrm{C}_{2} \mathrm{H}_{4}$ and $\mathrm{C}_{2} \mathrm{H}_{2}$. Also in 2008, Ağıral and co-authors reported on modelling of a DBD microreactor in propane, which nonetheless contained a thorough description of methane conversion pathways. ${ }^{142}$ Interestingly, the reactor converted the feed gas mainly into molecules with higher carbon numbers than propane, as opposed to thermal reactors mainly dissociating the propane. At similar conversion levels, the modelling was able to predict these selectivity trends satisfactorily. In 2009, Luche and co-authors reported modelling results of the production of syngas from methane-air mixtures in non-thermal plasma. ${ }^{143}$ The kinetic model considered only gas phase methane oxidation kinetics, excluding any plasma specific processes, whereas the discharge was accounted for as an additional heat term in the energy balance. An analysis was performed of the energy efficiency of the process. The most efficient reactor setup was found to have high flow rates, and mixtures with a low percentage of methane. However, higher conversion rates were found for lower flow rates, and with a higher percentage of methane. In 2012, Ravasio and Cavallotti published results on non-oxidative methane conversion modelling for various reactor types. ${ }^{38}$ Excitation reactions were not accounted for, however carbon deposition on the electrodes and detailed heat balances were considered in the OD models developed. Process parameters, such as specific energy input, were varied to determine their effect on conversion and efficiency. It was demonstrated that as specific energy increases, so too does the reactor efficiency, mainly attributed to the creation of thermal energy which accelerates dissociation. A reaction path analysis was performed to study the preferred pathways during a micro-discharge and the afterglow period (Figure 9), further used to develop a reduced reaction mechanism implemented in fluid dynamic simulations. In 2017, Khadir and co-authors presented zero-dimensional modelling results of a methane conversion process for the generation of hydrogen in a dielectric barrier discharge. ${ }^{144}$ The electric field, the control parameter of the zero-dimensional model, was generated using an equivalent circuit of the experimental setup. It was found that the dielectric capacitance is a parameter of particular importance in the conversion

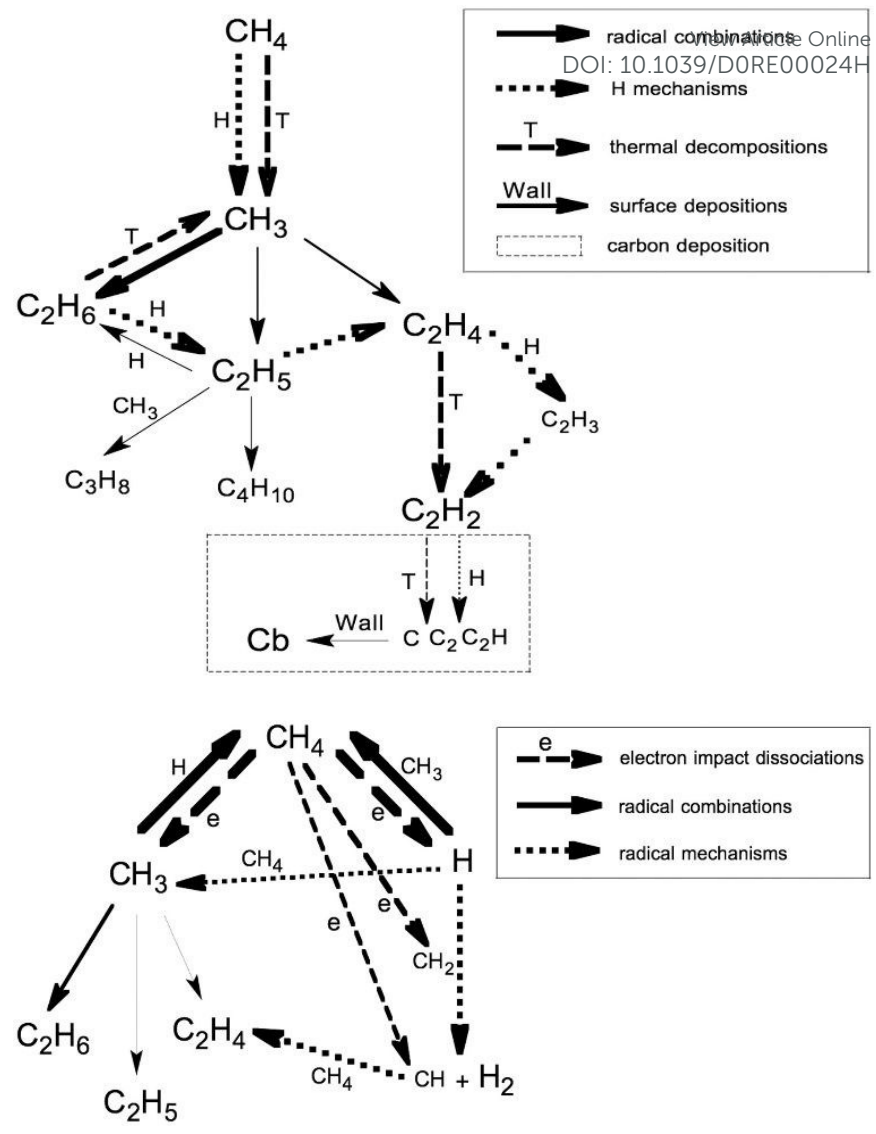

Figure 9. Main reaction pathways active in a micro-discharge during the potential pulse when the plasma is active (top) and when the discharge is off (bottom). Reprinted with permission from Ravasio and Cavallotti. ${ }^{38}$ Copyright (2012) Elsevier

process. Kudryashov and co-authors presented in 2018 modelling results in a dielectric barrier discharge for the conversion of methane under the presence of liquid water, the latter added to decrease the formation of carbon deposits on the electrodes. ${ }^{145} \mathrm{~A}$ method to obtain effective rate constants for the electron-molecule reactions was proposed by the authors to simplify the modelling. The same year, Wang and coauthors presented results on the propagation of the uncertainty in the measurements of the cross sections and rate coefficients used in a OD code. ${ }^{146}$ It was shown that the model uncertainty in the conversion percent can be as much as $33 \%$. Electron impact dissociation of $\mathrm{CH}_{4}$, recombination of $\mathrm{CH}_{3}$ and $\mathrm{H}$ to $\mathrm{CH}_{4}$ , and $\mathrm{C}_{2} \mathrm{H}_{6}$ production via three-body recombination of $\mathrm{CH}_{3}$ were revealed the most significant to the uncertainty of the conversion of $\mathrm{CH}_{4}$. Again in 2018, Toth et al. in order to capture the non-homogeneous filamentary nature of DBD proposed an alternative approach, wherein a 1D steady state plug flow reactor was modelled instead of the more commonly used $O D$ transient perfectly mixed reactor. ${ }^{42}$ Similar to other authors, ${ }^{29}$ the DBD spatiotemporal inhomogeneity was approximated through sub-sequences of pulses (equivalent to microdischarges) followed by afterglow periods subjected on a volume of gas, this sequence alternating though not temporally within a OD model but spatially within the 1D steady state model (see Figure 10). Finally, in 2019, Sun and Chen ${ }^{75}$ reported on results of zero-dimensional modelling of methane 
(a)
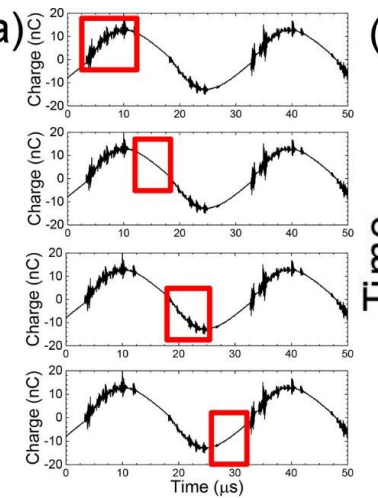

(b)

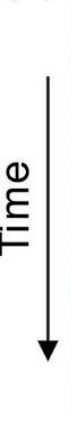

Real reactor

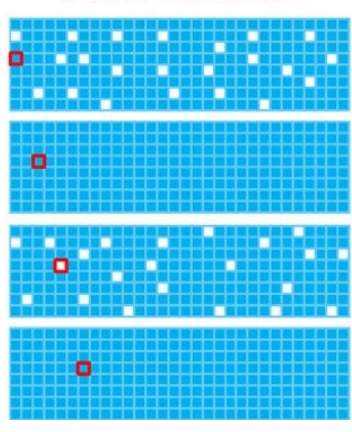

0-D model

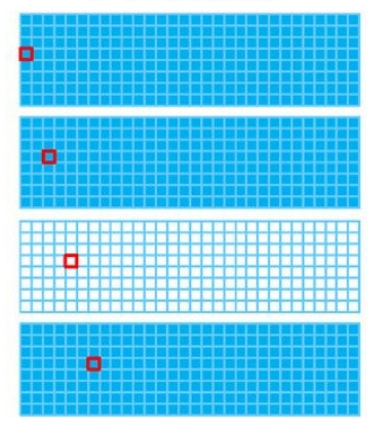

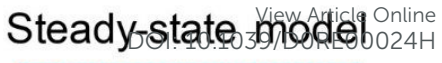

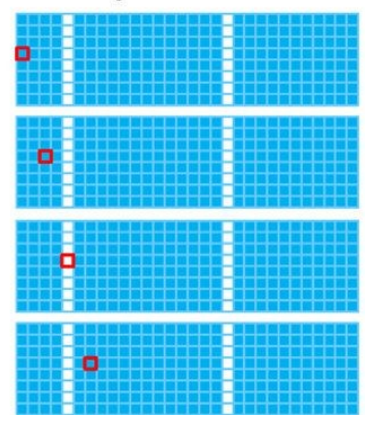

Figure 10. (a) Charge peaks obtained in a real DBD experiments, the spikes are happening during the filamentary micro-discharges when the smooth curve is the absence of microdischarge; (b) Comparison following an element of gas volume (red-edged square) in two different models. The white-filled squares correspond to elements of volume that are crossed by a micro-discharge. Adapted with permission from Toth et al. ${ }^{42}$ Copyright (2018) American Chemical Society

conversion in a radio frequency discharge. As commented previously, the work is characteristic in methane plasma modelling literature of being the only to date to consider the vibrationally excited states of methane as distinct species, finding that $31.4 \%$ of the conversion proceeded through these states (Figure 8).

\subsection{Fluid modelling}

Fluid models are often used to simulate the behaviour of methane plasmas. Fluid models are based on the assumption that certain components of a discharge, or the discharge as a whole, may be modelled as a reacting fluid, coupled to Maxwell's equations, in one, two, or three spatial dimensions. Different systems of fluid equations are used for different types of discharge. Dielectric barrier discharges, radio frequency discharges, and glow discharges, for example, may be modelled using equations of continuity of flux density of electrons and ions, coupled with Poisson's equation (as in e.g. ${ }^{37,44,53,98}$ ) Models of Corona discharge, ${ }^{147}$ and detailed models of streamers, ${ }^{148}$ include the physics of photo-ionisation. Arc discharges may be modelled using a fluid-based approach, for example, by using the magneto-hydrodynamic equations, written with the local thermodynamic equilibrium approximation. ${ }^{149}$ An advantage of fluid modelling is that for low species Knudsen numbers, near to Maxwellian energy distribution functions, and with accurate rate and transport coefficient data, the modelling may be quantitatively accurate. The disadvantage is that with increased dimensionality, there is an increased computational cost. Running simulations for a long period of time, or with very detailed reaction networks, is also computationally costly. A more detailed explanation of the fluid modelling approach can be found in Colonna and D'Angola. ${ }^{150}$

In the following recent findings from fluid modelling work of methane plasma reported in literature are discussed. In 2001, Bera and co-authors reported on 2D (axisymmetric, with the axis spanning the discharge gap between electrodes) modelling of low pressure methane RF plasma, used for plasma assisted chemical vapour deposition. ${ }^{50} \mathrm{~A}$ variety of surface processes were accounted for within a surface chemistry model to describe the carbon thin-film deposition/etch rate. The results showed significant radial variations in the discharge parameters, and that the plasma was electropositive. In 2001, Herrebout and co-authors published results of 1D fluid modelling, also on low pressure methane RF plasma. ${ }^{53}$ It was found that the plasma contained mainly $\mathrm{C}_{2} \mathrm{H}_{5}^{+}$ions, except for low pressures ( $<0.5$ Torr), where $\mathrm{CH}_{5}^{+}$ions were dominant. The higher hydrocarbons, $\mathrm{C}_{2} \mathrm{H}_{6}, \mathrm{C}_{2} \mathrm{H}_{4}, \mathrm{C}_{2} \mathrm{H}_{2}, \mathrm{C}_{3} \mathrm{H}_{8}$ were also present at high densities in the plasma. In 2006, Yarin and coauthors published modelling results of the growth of amorphous carbon layers on nano particles in low pressure methane plasma. ${ }^{51} \mathrm{~A}$ OD model of the bulk plasma was coupled to an analytic description of the boundary layer, and the surface of a nano particle. It was found that as the pressure increases, so too does the carbon deposition rate. The charging of the nano particles increased with their radius. In 2007, Naidis reported modelling results on a transient discharge in a stoichiometric mixture of atmospheric pressure methane and air. ${ }^{151}$ The discharge conditions capable of ignition of the mixtures were noted. The modelling demonstrated a rapid ignition by a discharge filament $\left(\tau_{i g n} \approx 10 \mu \mathrm{s}\right)$, as the plasma generated active species relevant for combustion. In 2011, De Bie and co-authors performed modelling of the conversion of pure atmospheric pressure methane by dielectric barrier discharge. ${ }^{37}$ As elaborated in previous sections, a detailed kinetic network was developed, describing excellently the ground state neutral gas chemistry, and was used in one dimensional fluid simulations. The results presented hydrogen and ethane as the main reaction products, which is consistent with experiments. The relevant reaction pathways were also discussed. In 2015, Pourali and Foroutan presented results of a space charge sheath fluid model coupled to a surface deposition model for a collection of nano particles at various locations in a sheath. ${ }^{56}$ It was found that the variation of the growth of nano particle size is based on the inhomogeneity of the plasma found in the space charge sheath, particularly of the concentration of the ions, as depicted in Figure 11. Finally, in 2018, Naidis demonstrated through one dimensional fluid modelling of streamer propagation, in various methane, nitrogen, and oxygen mixtures, that the streamer propagation velocity increases with heightened photon production. ${ }^{148}$ Mixtures that 


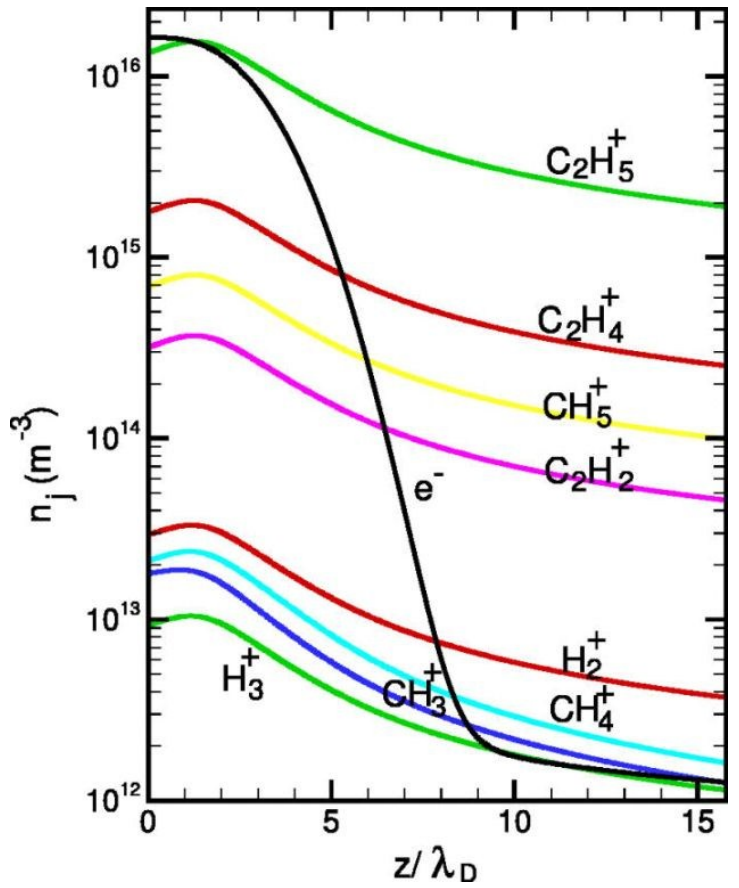

Figure 11. The spatial profile of the concentration of electrons and different ion species inside the plasma sheath. Reprinted with permission from Pourali and Foroutan ${ }^{56}$ Copyright (2015) AIP Publishing

absorb more photons, were shown to have a reduced streamer velocity.

\subsection{Particle In Cell - Monte Carlo Collision (PIC-MCC) modelling}

A less common approach used to simulate methane plasma is to use Particle In Cell - Monte Carlo Collision (PIC-MCC) modelling. In PIC-MCC codes, individual plasma species are treated as point macro-particles (ensembles of many, like, particles). These particles evolve in time by (often classical) equations of motion, coupled to Maxwell's equations, withbin a numerical cell. When the macro-particlescloselyozpprozecheazeh other, a Monte Carlo Collision code statistically models a particular reaction, based on, e.g. the velocities of the colliding species.

The work of Alexandrov and Schweigert is from the very few that have applied the method to model a methane plasma, specifically to simulate in two dimensions a radio frequency discharge. Depending on the voltage, two discharge regimes were identified, one at high voltages with an active sheath, where hot electrons are localised and reactions proceed preferentially, and one at lower voltages that is more uniform with reactions taking place across the entire reactor volume. The PIC-MCC approach has also been used to simulate a surface discharge on dielectric beads by Gao et al., however for an air plasma. ${ }^{152}$ Although not modelling a methane plasma, the work merits mention due to its direct relevance to plasma-catalysis, investigating mode transitions of filamentary discharges in packed bed DBD reactors. The ionisation and excitation rates obtained for nitrogen and oxygen are presented in Figure 12, where they are shown to be more pronounced on the surface of the dielectric beads.

One reason to use PIC-MCC codes for surfaces discharges is that the Knudsen number for the charged species is large, and so the assumptions used in the fluid approach break down. An advantage of using PIC-MCC codes is that few a priori assumptions are made, and so in principle, the codes can be very accurate. However, generally they are more computationally costly than fluid codes. Effective implementations in parallel computing architecture lead to codes capable or describing a very wide range of plasma phenomena. The review by Taccogna provides further details on the method. ${ }^{153}$

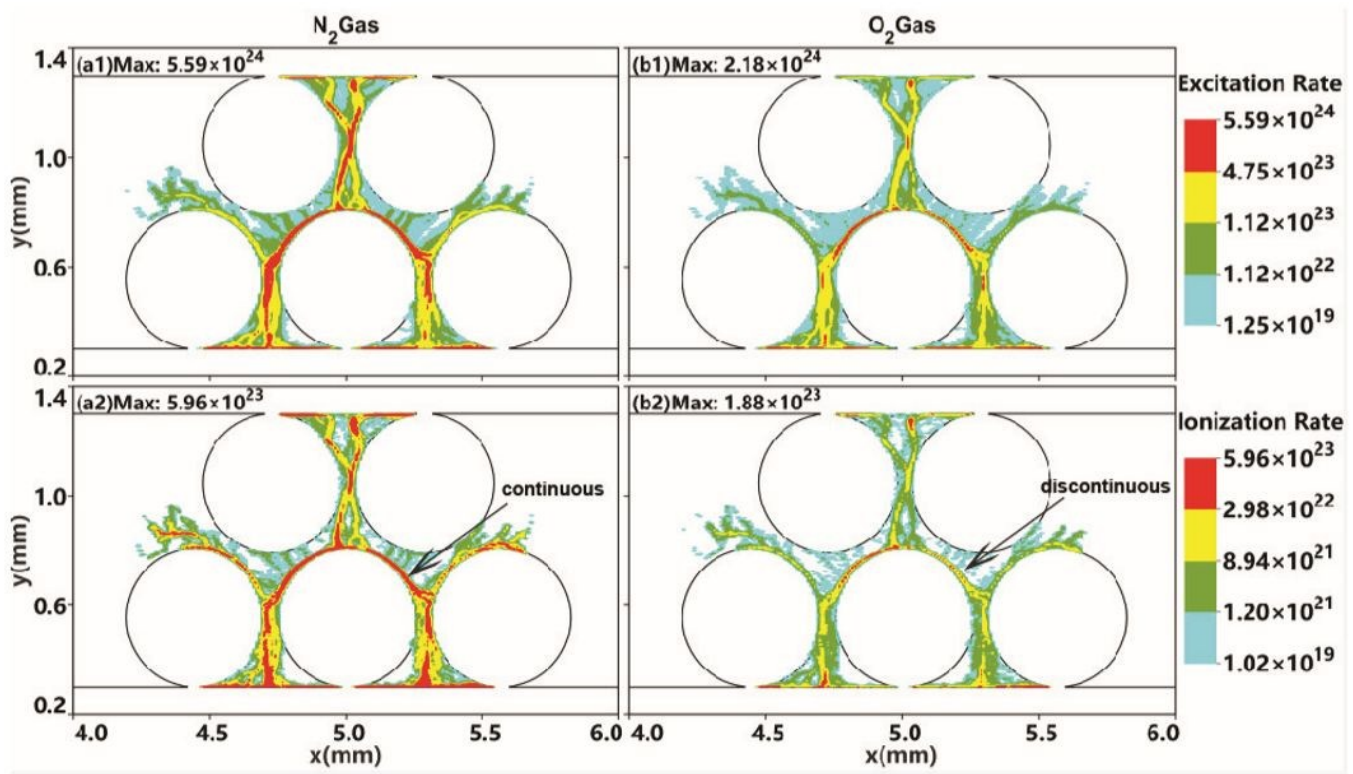

Figure 12. Electron impact excitation and ionisation rate, at $0.8 \mathrm{~ns}$, for a driving voltage of $20 \mathrm{kV}$. Nitrogen and oxygen components of an air discharge. Reprinted from Gao et al. 152 under the Creative Commons Attribution License 


\section{Plasma-catalysis modelling}

A variety of works, as presented in the previous sections, have indeed implemented surface chemistry models, coupled to gasphase fluid models of methane plasmas, however all concerned film deposition and etching applications related to semiconductors processing or particle coating. ${ }^{51,56,61,98}$ As such, the set of surface reactions considered aimed at describing the deposition rate of carbon on non-catalytic surfaces via the adsorption/desorption of radicals, ion-induced incorporation of these radicals in the growing film, direct ion incorporation in the film, etching and carbon sputtering. In plasma-catalysis, the adsorption of neutral molecules and their numerous follow-up surface reactions leading to the desorption of products, as encountered in thermal catalysis, is accompanied and potentially dominated by the interaction of the plasmagenerated radicals and excited species with the surface. The latter interactions could follow both Langmuir-Hinshelwood and Eley-Rideal mechanisms and would require an explicit description. Such elaborate modelling of the methane plasmacatalytic upgrading process, which combines the plasma phase dynamics and reactions, with catalytic surface reactions, has so far not been performed. ${ }^{154}$ Nonetheless, a variety of relevant approaches have been followed ranging from first principles methods to global modelling to describe specific effects in plasma-catalysis, a summary of which is presented below.

\subsection{Interaction of vibrationally excited states with catalyst surfaces}

As discussed previously, it has indeed been calculated that in non-thermal plasma discharges large amounts of the inputted energy are consumed in the vibrational excitation channel due to its large collision cross-section. ${ }^{17,75}$ In non-catalytic systems this energy is eventually largely lost through molecular collisions, dissipated as heat, leading to major energy losses that affect the process efficiency severely. ${ }^{17}$ As such, a typical consideration of the non-catalytic plasma chemistry modelling studies discussed above is the treatment of these excited states as only energy sinks. In the presence of a catalyst, though, depending on the relaxation rate of these vibrationally excited states, their higher internal energy in comparison with the ground state could lead to accelerated adsorption processes and overall catalytic performance enhancement. ${ }^{155}$ Indeed various experimental molecular beam studies have demonstrated that the dissociative chemisorption of methane on metal surfaces is characterised by strong mode specificity. ${ }^{156-167}$ For the same level of translational energy, the vibrationally excited states of methane have been shown to have higher initial sticking probabilities in comparison to ground state methane. Moreover, certain modes, particularly the $v 1$ and less so the $v 3$, were more effective in comparison to the ground state methane, when the latter was excited with the same amount of energy in translation.

From the above it clear that in the case of plasma-catalysis these excited state should be explicitly accounted for as distinct reactive species to be able to quantify their effect on catalytic rates and possibly elucidate if these are indeed the species leading to reported synergistic effects in plasma-catalysis.

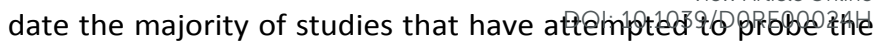
interaction of vibrationally excited methane with catalyst surfaces are first principle calculations based on quantum mechanics, primarily aiming to explain the above referenced molecular beam experimental results. Major findings from these theoretical works are summarised in the following.

In the work of Prasanna et al. a DFT based vibrational analysis along the minimum energy path (MEP) was performed to investigate the role of vibrational mode specificity in the dissociative chemisorption of methane on $N i(111) .{ }^{168}$ The transition state of the latter was found to resemble the product state, while the considerable elongation of the $C-H$ bond indicated the importance of the stretch modes over the bend modes. It was specifically found that the pre-excitation of the $v 1$ symmetric stretching mode would be approximately 3 times more efficient in promoting methane's adsorption than the preexcitation of the $v 3$ asymmetric stretching mode. Jackson and Nave, based on DFT calculations to locate the minimum energy path, used the Reaction Path Hamiltonian approach to study the dissociative chemisorption of methane on $N i(100) .{ }^{169}$ The vibrational excitation of methane was shown to significantly enhance the reaction when the molecule undergoes a transition to the ground state or to a lower energy vibrational state with the excess energy converted into motion along the reaction path. The $v 1$ mode was specifically shown to exhibit the highest reactivity on account of significant softening of the vibration taking place at the transition state. The authors extended their work on the $N i(111)$ surface arriving at similar conclusions, however in this case the reactivity of the $v 1$ and $v 3$ modes was found to be equivalent. ${ }^{170}$ Via extensive DFT calculations Jiang et al. constructed a global potential energy surface for methane interacting again with $N i(111) .{ }^{171}$ Quantum dynamic calculations of reactions dynamics showed that the excitation of specific $\mathrm{CH}_{4}$ vibrational modes of methane enhanced reactivity according to the order: $v 1>v 3>v 2>v 4$, with the two stretching modes further showing approximately the same enhancement. The calculated initial sticking probabilities $\left(S_{0}\right)$ for the various vibrational states were in good agreement with experimental results as seen in Figure 13.

The same authors further studied the reaction dynamics of the dissociative chemisorption of $\mathrm{CH}_{4}, \mathrm{CHD}_{3}$ and $\mathrm{CH}_{2} \mathrm{D}_{2}$ on the global potential energy surface from their previous work ${ }^{171}$ using Quasi-Classical Trajectory methods. ${ }^{172}$ Bond selectivity was demonstrated, as for $\mathrm{CHD}_{3}$ the excitation of the $C-H v 1$ mode enhanced the cleavage of the $C-H$ bond, whereas the dissociation of the $C-D$ bond was promoted by the excitation of the $C D_{3} v 2$ and $v 4$ modes. For $C H_{2} D_{2}$, excitation of one $C-H$ bond with two quanta was more effective than exciting both $\mathrm{C}-\mathrm{H}$ bonds with one quantum.

First principles MD simulations on the chemisorption of $C D_{3} H$ and $\mathrm{CH}_{3} \mathrm{D}$ on $\mathrm{Pt}(110)$ by Sacchi et al. ${ }^{173}$ showed that exciting the $C-H / C-D$ bond stretching in the $C D_{3} H / C H_{3} D$ isotopomers can promote selectively the chemisorption towards $C D_{3} / C H_{3}+H / D$, instead of $C D_{2} H_{/ C H} D+D / H$. In a follow-up work, Sacchi et al. ${ }^{174}$ used again MD simulations to study the chemisorption of $\mathrm{CH}_{4}$ on $\mathrm{Ni}(100)$. Agreeing well with 


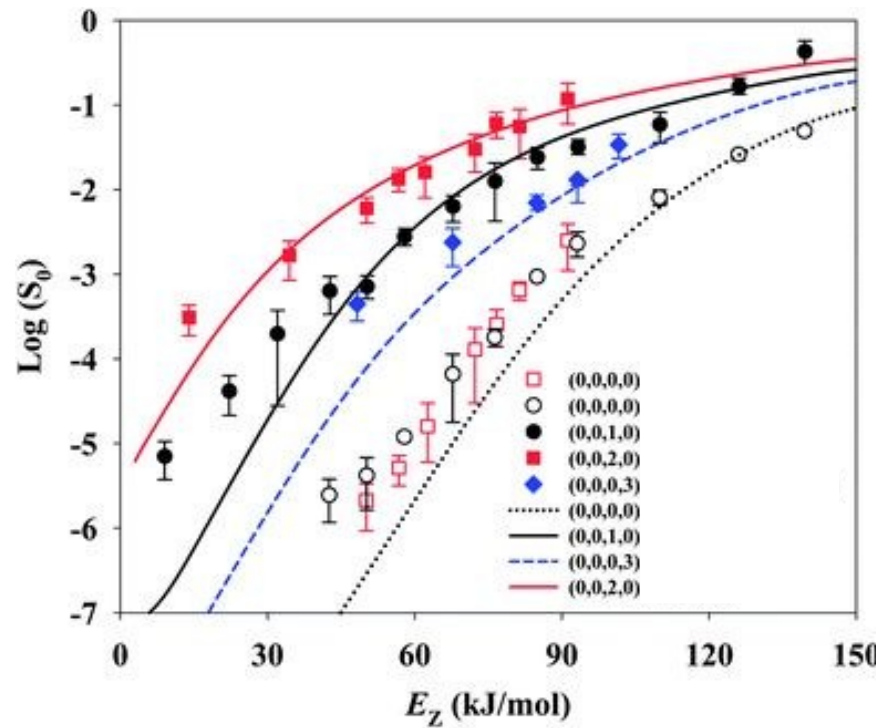

Figure 13. Comparison of calculated (lines from Jiang et al. ${ }^{171}$ ) and measured ( $\square$ and $\square$ from Bisson et al. ${ }^{160} \mathrm{O} \bullet$ from Smith et al. ${ }^{163}$ and $\bullet$ from Juurlink et al. ${ }^{162}$ ) initial sticking coefficients ( $S_{0}$ ) for various vibrational states of $\mathrm{CH}_{4}$ as a function of the collision energy. The notation $\left(n_{1}, n_{2}, n_{3}, n_{4}\right)$ refers to the vibrational quantum numbers for the $v_{1}, v_{2}, v_{3}$ and $v_{4}$ modes of $\mathrm{CH}_{4}$, respectively. Copyright (2013). Adapted from Jiang et al. ${ }^{171}$ with permission from The Royal Society of Chemistry.

the previously mentioned results, the reaction was shown to be mode-specific, with a high correlation between vibrational energy of the $v 1$ symmetric stretch mode and the energy localized in the reaction coordinate as methane approaches the transition state.

The interested reader is further referred to the review by Jiang et al. ${ }^{175}$ that covers in depth recent findings from quantum dynamics studies on the mode specificity and bond selectivity in the chemisorption of polyatomic molecules, namely $\mathrm{CH}_{4}$ and $\mathrm{H}_{2} \mathrm{O}$, on transition metal surfaces.

\subsection{Interaction of radicals with catalyst surfaces}

As commented above, the electron energy distribution function affects directly the amount and type of radicals generated in the plasma. These radicals can also proceed to react op the featalyst

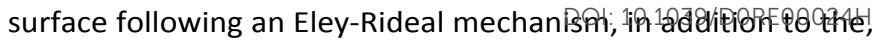
typical for the majority of thermal-catalytic processes, Langmuir-Hinshelwood mechanism for molecules. Radicalssurface interactions are highly complex, leading either to the associative desorption of closed shell molecules or to the formation of open shell molecules trapped as adsorbates. ${ }^{18,176}$ Both the former and the latter participate in the plasma phase or surface reaction networks respectively, further affecting the process.

The adsorption of $\mathrm{CH}_{x}$ radicals on Nickel surfaces and their subsequent reactions were investigated via reactive molecular dynamics simulations by Neyts and co-workers over $\mathrm{Ni}(111)$ and $N i(100)$ at the relevant for non-thermal plasma-catalysis temperature of $400 \mathrm{~K} .{ }^{177}$ In line with similar studies in the frame of thermal-catalysis, the $N i(100)$ surface was found to be the most active both in terms of radicals' adsorption probability and of their follow-up dissociation reactivity. Interestingly, the cleavage of $C-H$ bonds of adsorbed radicals was found to be taking place on $\mathrm{Ni}(111)$ principally via the abstraction of a $H$ atom by a colliding gas phase radical, according to an EleyRideal type of reaction. Conversely, on the $N i(100)$ a direct dissociation of the $C-H$ bond was dominant. Furthermore, the formation of $\mathrm{C}_{2}$ species was found to be primarily driven by the impact of $\mathrm{CH}_{2}$ and $\mathrm{CH}$ radicals. In the follow-up work of the authors, the effect of temperature on $\mathrm{CH}_{x}$ radicals' adsorption and reaction over $\mathrm{Ni}(111)$ was also investigated via reactive $\mathrm{MD}$, in relevance to warm plasmas combined with catalysis. ${ }^{178}$ Temperatures above $1000 \mathrm{~K}$ were seen to promote $\mathrm{H}_{2}$ production, but at the same time carbon diffusion in the subsurface layers of $N i$, as illustrated in Figure 14 . The work of the authors was further expanded to consider various $\mathrm{Ni}$ surfaces including $N i(111), N i(100)$, step-edged $N i(111)$, polycrystalline and amorphous at the wide temperature range of 400 to $1600 \mathrm{~K} .{ }^{179}$ Higher temperatures were seen to promote $\mathrm{C}-\mathrm{H}$ bond breaking upon collision of the radicals, the effect being more pronounced on $\mathrm{CH}_{3}$, then $\mathrm{CH}_{2}$ and less so on $\mathrm{CH}$. The $N i(100)$ and stepped $N i(111)$ were the most reactive, although, at the higher temperatures, differences became less

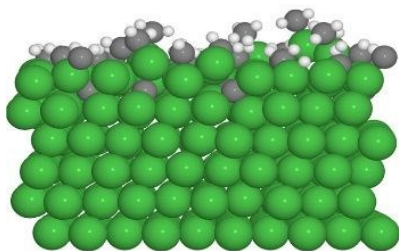

$400 \mathrm{~K}$

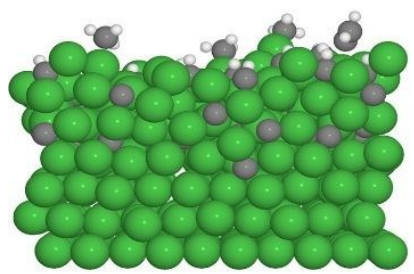

$1200 \mathrm{~K}$

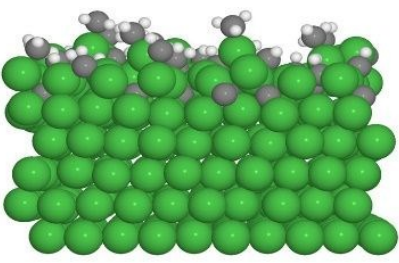

$800 \mathrm{~K}$

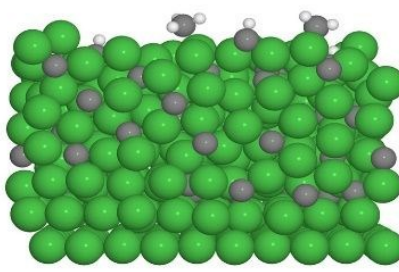

$1400 \mathrm{~K}$

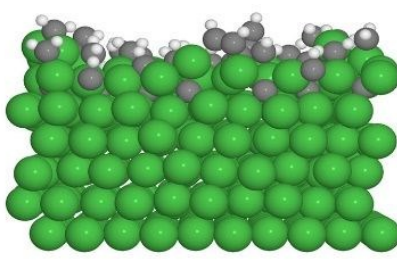

$1000 \mathrm{~K}$

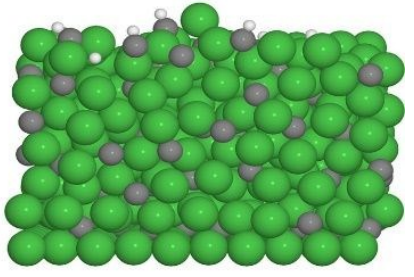

$1600 \mathrm{~K}$

Figure 14. Side view of the $\mathrm{Ni}(111)$ surface after 250 consecutive $\mathrm{CH}_{2}$ impacts at different temperatures. The green spheres correspond to the nickel atoms, the gray spheres to the $\mathrm{C}$-atoms and the white spheres to the $\mathrm{H}$-atoms. Reprinted with permission from Somers et al. ${ }^{178}$ Copyright (2013) Elsevier 
evident due to the surfaces losing their crystallinity from the progressive diffusion of $C$ atoms into subsurface layers.

Density functional theory calculations of $\mathrm{CH}_{4}$ and $\mathrm{C}_{2}$ hydrocarbons reactions on metal surfaces have been the subject of numerous studies due their relevance to, conventional, high temperature catalytic steam and dry reforming. Within the scope of the current review, the work of Shirazi et al. ${ }^{180}$ investigated the surface reactions of $\mathrm{CH}_{x}$ fragments on $N i(111)$. The authors showed that the abundance of $H$ atoms on the catalyst surface facilitated the hydrogenation of $\mathrm{CH}_{x}$ species but could also effectively promote the desorption of closed shell molecules, such as ethylene.

While evidently highly relevant to plasma catalysis, it should be noted that the above studies and those discussed in Section 5.1, focused on adsorption or surface mechanistic details, not considering any features of the plasma phase and the associated chemistry.

\subsection{Global plasma-catalysis kinetic studies}

Global kinetic studies have been carried out to deduce overall mechanistic features of plasma-catalytic reactions in comparison to the respective thermal-catalytic. Nozaki et al. ${ }^{17,181}$ analysed forward methane conversion rates via the Arrhenius Plot method for the case of thermal-catalytic and hybrid plasma-thermal-catalytic methane steam reforming, based on previous experimental results from the same authors. ${ }^{182}$ The apparent activation energy determined for both reactors was interestingly found to be the same in both the reaction- and diffusion-limited regimes, whereas significantly larger pre-exponential factors were calculated for the plasma case (Figure 15). The results suggested a reaction mechanism where methane activation remains the rate limiting step also under plasma-catalysis, with, however, the vibrational excitation of methane enhancing its dissociative chemisorption on the catalyst.

Zheng et al. analysed via the Arrhenius Plot method experimental results of plasma-catalytic methane dry reforming

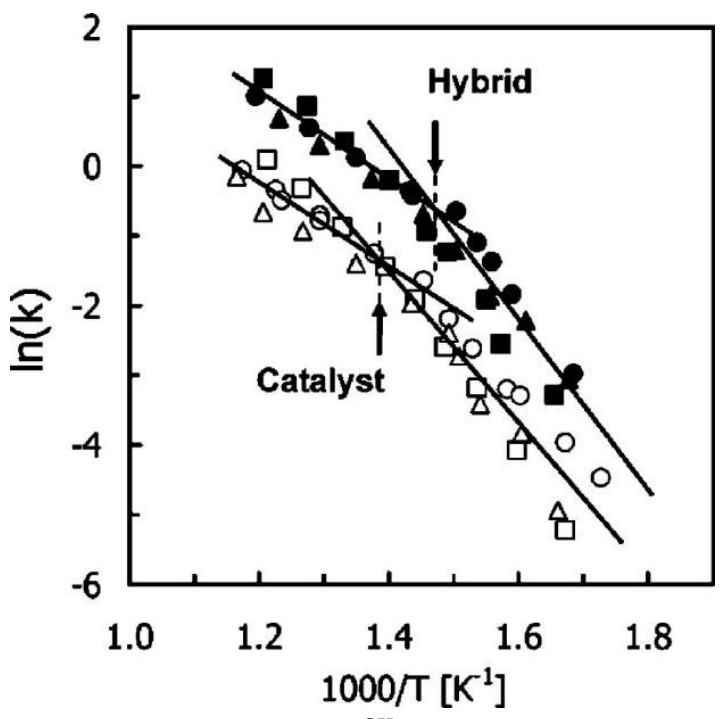

Figure 15. Arrhenius plot for the forward $\boldsymbol{C H}_{\mathbf{4}}$ reaction rate constant. ( $\mathrm{O}$ and $\bullet$ ) GHSV = $18,000 \mathrm{~h}^{-1}, \mathrm{~S} / \mathrm{C}=1 ;(\square$ and $\boldsymbol{\square}) \mathrm{GHSV}=18,000 \mathrm{~h}^{-1}, \mathrm{~S} / \mathrm{C}=3$; and $(\triangle$ and $\mathbf{\Delta}) \mathrm{GHSV}=10,800$ $h^{-1}, S / C=1$. Diffusion limited regime evident by the change in the slope at higher temperatures. Reprinted with permission from Nozaki et al. ${ }^{181}$ Copyright (2007) American Chemical Society assuming power-law rate equations for the conversion of the reactants. ${ }^{183}$ The authors estimated DQppparefit/DGeEPatäbin barriers both for $\mathrm{CH}_{4}$ and $\mathrm{CO}_{2}$ conversion in the range of 25 to $30 \mathrm{~kJ} / \mathrm{mol}$, much lower to the typically reported values in thermal-catalytic studies. Nonetheless, this difference was mainly attributed to the high dispersion of metallic nickel in the structure of the core-shell $\mathrm{Ni}-\mathrm{La}_{2} \mathrm{O}_{3} @ \mathrm{SiO}_{2}$ catalyst used for the experimental study. The same authors developed a global kinetic model for plasma-catalytic methane dry reforming, assuming that i) gas molecules in the bulk can convert to radicals via electron collisions, ii) radicals and molecules can convert to products on the catalyst, and iii) radicals can terminate towards bulk gas molecules. ${ }^{184} \mathrm{~A}$ simple first order kinetic expression was obtained, linking input power and residence time with total conversion, which allowed the comparison of reactivities of $\mathrm{CH}_{4}$ and $\mathrm{CO}_{2}$ over the catalyst, finding the former to be more selective towards products formation than the latter. Also for methane dry reforming, Kim et al. ${ }^{185}$ assumed power law and Langmuir Hinshelwood expressions to describe rate data obtained during thermalcatalytic and plasma-thermal-catalytic experiments, respectively. Non-Arrhenius behaviour was observed during analysis of the plasma-catalytic results prompting the authors to propose the below modified Arrhenius expression, which, as with the work of Zheng et al., ${ }^{184}$ linked conversion to input power and residence time:

$$
k_{\text {plasma }- \text { cat }}=k_{\text {plasma }}^{\prime}-\text { cat } \times e^{-\frac{E_{B A R R I E R}}{\frac{1}{\text { total gas flow rate }} \times \text { power }_{D B D}}}
$$

Much better correlation with the experimental results was obtained via this method. Apparent activation barriers estimated were much lower than the equivalent thermal catalytic, which the authors associated to the rate determining step being the dissociation of, higher internal energy, vibrationally excited methane already adsorbed on the catalyst.

\section{Conclusions}

In order to unravel the plasma-catalysis system for methane upgrading, the incorporation of the surface chemistry of catalysts into plasma fluid models needs to be prioritised. The dominant mechanisms in the process, be they catalytic or plasma based, or some synergistic combination, can be analysed and optimised this way. As reviewed, in most plasmacatalysis modelling studies plasma phase reactions were omitted highlighting the need for a fundamental kinetic approach to be taken, where plasma chemistry is explicitly coupled with surface kinetics.

For the case of catalytic reactions, microkinetic modelling is proposed as a most suitable approach to enhance the understanding and enable the optimisation of plasmaenhanced heterogeneous catalytic reaction systems, based on the demonstrated ability of the method in thermal catalysis of accelerating catalyst and process design via reaction mechanism elucidation and catalyst discrimination. ${ }^{186}$ Elaborate microkinetic models have been developed for

This journal is (C) The Royal Society of Chemistry 20xx 
example for high temperature oxidative coupling of methane aiming at describing the complex interplay between homogeneous and heterogeneous catalytic chemistries, comprehensively accounting for gas-phase reactions between radicals, Langmuir-Hinshelwood reactions of surface species and the interaction of molecules and radicals with the catalytic surface via Eley-Rideal reactions. ${ }^{187}$

To achieve the above, a route similar to that followed in thermal catalysis will need to be taken, namely combining the outputs of density functional theory calculations and microkinetics. The DFT calculations allow microscopic properties of catalytic systems such as the binding energies of involved surface species to be obtained. The latter can further feed into microkinetic models of surface reactions linking the quantum-chemical information with the macroscopic behaviours of the actual systems. ${ }^{186}$ There are indeed DFT studies on plasma specific species being already carried out, such as the reviewed works on the vibrational modes of methane, however the latter should be expanded to the multitude of species encountered within a methane plasma.

The above implies that better insights on the elementary processes that occur in the plasma phase must also be brought, so that plasma phase kinetic models are improved. For a comprehensive heterogeneous catalytic microkinetic model to be developed, able to account for the interactions of all plasma species with the catalyst surface, these species need to be explicitly considered within the plasma phase. The description of excited states in methane plasma models is for now poorly investigated. Very recently there was a first attempt to account for the vibrational modes of methane as distinct species. ${ }^{75} \mathrm{It}$ is indeed critical that the reactivity and relaxation of these excited states is modelled explicitly in the plasma phase. A similar approach should be followed for the electronically excited states, which is a model feature presently missing in methane plasma literature. Fundamental research into the rates and cross sections in order to reduce the experimental uncertainties would also result in more accurate models.

In the case of fluid modelling, where Poisson's equation is solved, the consideration of ions is extremely important as their charge changes the field. For this type of modelling, the greatest level of details on ions and their processes is advised. 3D fluid modelling could be performed to investigate the role of the filamentary nature of some dielectric barrier discharges on conversion rates. This approach would complement the literature on $O D$ modelling, as the latter lacks the capability of modelling the filaments, and the $3 \mathrm{D}$ modelling lacks the capability to use such sophisticated reaction networks.

In a fully consistent model, the important role of coking on a catalyst should be investigated, although this has proven to be a complex task even in the case of thermal catalysis. In this regard, the knowledge obtained from studies in plasma literature on chemical vapor deposition and thin-film growth on non-catalytic substrates can prove beneficial to make advancements in the modelling.

Lastly, the interaction of charged species with the catalyst surface would need to be elaborated to fully understand the complex and possibly synergistic plasma-catalyst interactions.
Quantum chemical models based on density functional theory have recently appeared in literature stedyingo the 0 effect 2 of negative charging of a catalyst surface in relation to the plasma catalytic activation of $\mathrm{CO}_{2}{ }^{188}$ so it is imperative that such advancements are also made in the methane plasma field.

Progress in all modelling aspects and improvements on relevant methodologies applied in other plasma-catalytic systems or even research fields will obviously need to be combined with similar developments in the experimental techniques used to study such systems, resulting in building a comprehensive picture of plasma-catalytic methane upgrading systems, enabling its optimisation.

\section{Conflicts of interest}

There are no conflicts to declare.

\section{Acknowledgements}

The work was supported by the UK Engineering and Physical Sciences Research Council (EPSRC) New Investigator Award, grant no. EP/R031800/1.

\section{Notes and references}

1 W. Cho, Y. Baek, H. Pang and Y. C. Kim, Korean J. Chem. Eng., 1998, 15, 500-504.

2 A. Marafee, C. Liu, G. Xu, R. Mallinson and L. Lobban, Ind. Eng. Chem. Res., 1997, 36, 632-637.

3 C. Liu, A. Marafee, R. Mallinson and L. Lobban, Appl. Catal. Gen., 1997, 164, 21-33.

4 C.-J. Liu, R. Mallinson and L. Lobban, Appl. Catal. Gen., 1999, 178, 17-27.

5 J. R. Fincke, R. P. Anderson, T. Hyde, B. A. Detering, R. Wright, R. L. Bewley, D. C. Haggard and W. D. Swank, Plasma Chem. Plasma Process., 2002, 22, 105-136.

6 F. Saleem, J. Kennedy, U. H. Dahiru, K. Zhang and A. Harvey, Chem. Eng. Process. - Process Intensif., 2019, 142, 107557.

7 Y. Yang, Plasma Chem. Plasma Process., 2003, 23, 283-296.

8 X.-S. Li, A.-M. Zhu, K.-J. Wang, Y. Xu and Z.-M. Song, Catal. Today, 2004, 98, 617-624.

9 C. Xu and X. Tu, J. Energy Chem., 2013, 22, 420-425.

10 L. Bromberg, D. R. Cohn, A. Rabinovich and N. Alexeev, Int. J. Hydrog. Energy, 1999, 24, 1131-1137.

11 M. Kraus, W. Egli, K. Haffner, B. Eliasson, U. Kogelschatz and A. Wokaun, Phys Chem Chem Phys, 2002, 4, 668-675.

12 Q. Wang, Y. Cheng and Y. Jin, Catal. Today, 2009, 148, 275282.

13 Z. Sheng, S. Kameshima, K. Sakata and T. Nozaki, in Plasma Chemistry and Gas Conversion, eds. N. Britun and T. Silva, IntechOpen, 2018.

14 M. A. Malik, S. A. Malik and X.-Z. Jiang, J. Nat. Gas Chem., 1999, 8, 166-178+180.

15 A. I. Babariotskii, M. A. Deminskii, A. I. Demkin, V. K. Zhivotov, B. V. Potapkin, S. V. Poteknin, V. D. Rusanov, E. I. Ryazantsev and C. Etievan, High Energy Chem., 1999, 33, 45-51.

16 J. Zhang, J. K. S. Wan, L. Cao and Y. Yang, Chin. J. Catal., 1999, 20, 50 .

17 T. Nozaki and K. Okazaki, Catal. Today, 2013, 211, 29-38. 
18 H. L. Chen, H. M. Lee, S. H. Chen, Y. Chao and M. B. Chang, Appl. Catal. B Environ., 2008, 85, 1-9.

19 M. Scapinello, E. Delikonstantis and G. D. Stefanidis, Chem. Eng. Process. Process Intensif., 2017, 117, 120-140.

20 H. Puliyalil, D. Lašič Jurković, V. D. B. C. Dasireddy and B. Likozar, RSC Adv., 2018, 8, 27481-27508.

21 G. SriBala, D. Michiels, C. Leys, K. M. Van Geem, G. B. Marin and A. Nikiforov, J. Clean. Prod., 2019, 209, 655-664.

22 U. Kogelschatz, Plasma Chem. Plasma Process., 2003, 23, 1-46.

23 E. C. Neyts, Plasma Chem. Plasma Process., 2016, 36, 185-212.

24 T. Pham Huu, S. Gil, P. Da Costa, A. Giroir-Fendler and A. Khacef, Catal. Today, 2015, 257, 86-92.

25 M. R. Rahimpour, A. Jahanmiri, M. Mohamadzadeh Shirazi, N. Hooshmand and H. Taghvaei, Chem. Eng. J., 2013, 219, 245253.

26 M. Taheraslani and H. Gardeniers, Energies, , DOI:10.3390/en13020468.

27 Y. Zeng, X. Zhu, D. Mei, B. Ashford and X. Tu, Catal. Today, 2015, 256, 80-87.

28 A. Bogaerts, C. De Bie, R. Snoeckx and T. Kozák, Plasma Process. Polym., 2017, 14, 1600070.

29 R. Snoeckx, R. Aerts, X. Tu and A. Bogaerts, J. Phys. Chem. C, 2013, 117, 4957-4970.

30 W. Wang, R. Snoeckx, X. Zhang, M. S. Cha and A. Bogaerts, J. Phys. Chem. C, 2018, 122, 8704-8723.

31 A. Janeco, N. R. Pinhão and V. Guerra, J. Phys. Chem. C, 2015, 119, 109-120.

32 C. De Bie, J. van Dijk and A. Bogaerts, J. Phys. Chem. C, 2015, 119, 22331-22350.

33 C. Li, B. Kuan, W. J. Lee, N. Burke and J. Patel, Chem. Eng. Sci., 2018, 187, 189-199.

34 G. Li, M. Qian, J. Kang, S. Liu, C. Ren, J. Zhang and D. Wang, Jpn. J. Appl. Phys., 2018, 57, 096204.

35 Q. Chen, J. Sun and X. Zhang, Chin. J. Chem. Eng., 2018, 26, 1041-1050.

36 Y. Yang, Plasma Chem. Plasma Process., 2003, 23, 327-346.

37 C. De Bie, B. Verheyde, T. Martens, J. van Dijk, S. Paulussen and A. Bogaerts, Plasma Process. Polym., 2011, 8, 1033-1058.

38 S. Ravasio and C. Cavallotti, Chem. Eng. Sci., 2012, 84, 580590.

39 T. Kovács, Plasma Chem. Plasma Process., 2010, 30, 207-212.

40 A. Indarto, J.-W. Choi, H. Lee and H. Keun Song, J. Nat. Gas Chem., 2005, 14, 13-21.

41 A. Indarto, N. Coowanitwong, J.-W. Choi, H. Lee and H. K. Song, Fuel Process. Technol., 2008, 89, 214-219.

42 J. R. Toth, X. Shen, D. J. Lacks and R. M. Sankaran, Ind. Eng. Chem. Res., 2018, 57, 6048-6056.

43 T. Nozaki, N. Muto, S. Kado and K. Okazaki, Catal. Today, 2004, 89, 57-65.

44 A. Gadoum, D. Benyoucef and M. H. A. Lahoual, in Renewable Energy for Smart and Sustainable Cities, ed. M. Hatti, Springer International Publishing, Cham, 2019, pp. 516-522.

45 H. Bahador and N. Pourali, Phys. Plasmas, 2019, 26, 013502.

46 Y. Zhao, C. Wang, L. Li, L. Wang and J. Pan, Phys. Plasmas, 2018, 25, 033504.

47 H. Zhang, W. Wang, X. Li, L. Han, M. Yan, Y. Zhong and X. Tu, Chem. Eng. J., 2018, 345, 67-78.

48 R. Snoeckx, M. Setareh, R. Aerts, P. Simon, A. Maghari and A. Bogaerts, Int. J. Hydrog. Energy, 2013, 38, 16098-16120.

49 J. Legrand, A. Diamy, R. Hrach and V. Hrachová, Vacuum, 1997, 48, 671-675.
50 J.-C. Legrand, A.-M. Diamy, R. Hrach, V. Hrachová and reacctib Vacuum, 1998, 50, 491-495. DOI: 10.1039/DORE00024H

51 A. L. Yarin, B. Rovagnati, F. Mashayek and T. Matsoukas, J. Appl. Phys., 2006, 99, 064310.

52 K. Tachibana, M. Nishida, H. Harima and Y. Urano, J. Phys. Appl. Phys., 1984, 17, 1727-1742.

53 D. Herrebout, A. Bogaerts, M. Yan, R. Gijbels, W. Goedheer and E. Dekempeneer, J. Appl. Phys., 2001, 90, 570-579.

54 M. Masi, C. Cavallotti and S. Carrà, Chem. Eng. Sci., 1998, 53, 3875-3886.

55 A. von Keudell and W. Möller, J. Appl. Phys., 1994, 75, 77187727.

56 N. Pourali and G. Foroutan, Phys. Plasmas, 2015, 22, 103514.

57 S. F. Yoon, K. H. Tan, Rusli and J. Ahn, J. Appl. Phys., 2001, 91, 40-47.

58 I. Hinkov, K. Pashova and S. Farhat, Diam. Relat. Mater., 2019 93, 84-95.

59 K. Tanaka and N. Mutsukura, Plasma Chem. Plasma Process., 1999, 19, 217-227.

60 N. Mutsukura, S. Inoue and Y. Machi, J. Appl. Phys., 1992, 72, 43-53.

61 K. Bera, B. Farouk and P. Vitello, J. Phys. Appl. Phys., 2001, 34, 1479-1490.

62 K. Pashova, I. Hinkov, X. Aubert, S. Prasanna, F. Bénédic and S. Farhat, Plasma Sources Sci. Technol., 2019, 28, 045001.

63 U. Sharma and S. C. Sharma, Phys. Plasmas, 2018, 25, 103509.

64 H. Mohammadzadeh, N. Pourali and Z. Ebadi, Phys. Plasmas, 2018, 25, 033503.

65 M. Qian, G. Li, J. Kang, S. Liu, C. Ren, J. Zhang and D. Wang, Phys. Plasmas, 2018, 25, 013519.

66 I. V. Adamovich, M. Nishihara, I. Choi, M. Uddi and W. R. Lempert, Phys. Plasmas, 2009, 16, 113505.

67 Adamovich Igor V., Li Ting and Lempert Walter R., Philos. Trans. R. Soc. Math. Phys. Eng. Sci., 2015, 373, 20140336.

68 J. K. Lefkowitz, P. Guo, A. Rousso and Y. Ju, Philos. Trans. R. Soc. Math. Phys. Eng. Sci., 2015, 373, 20140333.

69 A. C. DeFilippo and J.-Y. Chen, Combust. Flame, 2016, 172, 3848.

70 D. Breden, L. L. Raja, C. A. Idicheria, P. M. Najt and S. Mahadevan, J. Appl. Phys., 2013, 114, 083302.

71 D. Breden, C. A. Idicheria, S. Keum, P. M. Najt and L. L. Raja, IEEE Trans. Plasma Sci., 2019, 47, 410-418.

72 Q. Chen, X. Yang, J. Sun, X. Zhang, X. Mao, Y. Ju and B. E. Koel, Plasma Chem. Plasma Process., 2017, 37, 1551-1571.

73 N. Tsolas, R. A. Yetter and I. V. Adamovich, Combust. Flame, 2017, 176, 462-478.

74 G. LI, M. QIAN, S. LIU, H. CHEN, C. REN and D. WANG, Plasma Sci. Technol., 2017, 20, 014004.

75 J. Sun and Q. Chen, J. Energy Chem., 2019, 39, 188-197.

76 M. A. Deminskii, I. V. Chernysheva, S. Ya. Umanskii, M. I. Strelkova, A. E. Baranov, I. V. Kochetov, A. P. Napartovich, T. Sommerer, S. Saddoughi, J. Herbon and B. V. Potapkin, Russ. J. Phys. Chem. B, 2013, 7, 410-423.

77 J. DENG, L. HE, X. LIU and Y. CHEN, Plasma Sci. Technol., 2018, 20, 125502.

78 Z. Eckert, N. Tsolas, K. Togai, A. Chernukho, R. A. Yetter and I. V. Adamovich, J. Phys. Appl. Phys., 2018, 51, 374002.

79 J. Han and H. Yamashita, Combust. Flame, 2014, 161, 20642072.

80 I. N. Kosarev, N. L. Aleksandrov, S. V. Kindysheva, S. M. Starikovskaia and A. Yu. Starikovskii, Combust. Flame, 2008, 154, 569-586. 
81 R. Sanchez-Gonzalez, Y. Kim, L. A. Rosocha and S. Abbate, IEEE Trans. Plasma Sci., 2007, 35, 1669-1676.

82 D. I. Slovetskii, Yu. A. Mankelevich, S. D. Slovetskii and T. V. Rakhimova, High Energy Chem., 2002, 36, 44-52.

83 S. Wang, J. Yu, W. Cheng, Y. Ma, R. Zheng, D. Huang and Q. Wei, Chem. Phys. Lett., 2019, 730, 399-406.

84 B. I. MIN, D. K. DINH, D. H. LEE, T. H. KIM and S. CHOI, Plasma Sci. Technol., 2019, 21, 064005.

85 B. Chen, H. Wang, Z. Wang, J. Han, A. Alquaity, H. Wang, N. Hansen and M. Sarathy, DOI:10.1016/j.combustflame.2019.01.009.

86 J. Kang, M. Qian, G. Li, S. Liu, C. Ren, J. Zhang and D. Wang, Phys. Plasmas, 2018, 25, 093508.

87 E. Gogolides, C. Buteau, A. Rhallabi and G. Turban, J. Phys. Appl. Phys., 1994, 27, 818-825.

88 A. A. Fridman, Plasma chemistry, Cambridge University Press, Cambridge, First paperback edition., 2012.

89 G. J. M. Hagelaar and L. C. Pitchford, Plasma Sources Sci. Technol., 2005, 14, 722-733.

90 Dyatko N.A., Kochetov I.V., Napartovich A.P. and Sukharev A.G., EEDF: The software package for calculations of Electron Energy Distribution Function, .

91 A. Tejero-del-Caz, V. Guerra, D. Gonçalves, M. L. da Silva, L. Marques, N. Pinhão, C. D. Pintassilgo and L. L. Alves, Plasma Sources Sci. Technol., 2019, 28, 043001.

92 P. Koelman, S. Heijkers, S. Tadayon Mousavi, W. Graef, D. Mihailova, T. Kozak, A. Bogaerts and J. van Dijk, Plasma Process. Polym., 2017, 14, 1600155.

93 H. M. Anderson, J. A. Merson and R. W. Light, IEEE Trans. Plasma Sci., 1986, 14, 156-164.

94 R. Girard, J. B. Belhaouari, J. J. Gonzalez and A. Gleizes, J. Phys. Appl. Phys., 1999, 32, 2890-2901.

95 R. A. Gottscho and C. E. Gaebe, IEEE Trans. Plasma Sci., 1986, 14, 92-102.

96 D. K. Davies, L. E. Kline and W. E. Bies, J. Appl. Phys., 1989, 65, 3311-3323.

97 E. Gogolides, D. Mary, A. Rhallabi and G. Turban, Jpn. J. Appl. Phys., 1995, 34, 261.

98 T. Farouk, B. Farouk, A. Gutsol and A. Fridman, J. Phys. Appl. Phys., 2008, 41, 175202.

99 M. Scapinello, E. Delikonstantis and G. D. Stefanidis, Chem. Eng. J., 2019, 360, 64-74.

100 M. S. Bak, H. Do, M. G. Mungal and M. A. Cappelli, Combust. Flame, 2012, 159, 3128-3137.

101 A. T. Droege and P. C. Engelking, Chem. Phys. Lett., 1983, 96, 316-318.

102 S. Y. T. van de Meerakker, N. Vanhaecke, M. P. J. van der Loo, G. C. Groenenboom and G. Meijer, Phys. Rev. Lett., 2005, 95, 013003.

103 B. R. Turner, J. A. Rutherford and D. M. J. Compton, J. Chem. Phys., 1968, 48, 1602-1608.

104 M. Molteni and A. Donazzi, Chem. Eng. Sci., 2020, 212, 115340.

105 T. Nozaki, N. Muto, S. Kadio and K. Okazaki, Catal. Today, 2004, 89, 67-74.

106 Y. Gao, S. Zhang, H. Sun, R. Wang, X. Tu and T. Shao, Appl. Energy, 2018, 226, 534-545.

107 T. L. Van Surksum, J. M. Blechle and E. R. Fisher, J. Vac. Sci. Technol. A, 2018, 36, 041302.

108 T. Kozák and A. Bogaerts, Plasma Sources Sci. Technol., 2014, 23, 045004
109 S. H. Moreno, A. I. Stankiewicz and G. D. Stefanidis, React. Chem. Eng., 2019, 4, 2108-2116. DOI: 10.1039/DORE00024H

110 J. T. Yardley and C. B. Moore, J. Chem. Phys., 1968, 49, 11111125.

111 W. L. Morgan, Plasma Chem Plasma Process.

112 Puech database, https://fr.lxcat.net, (accessed 3 June 2019).

113 Hayashi database, https://fr.lxcat.net, (accessed 3 June 2019).

114 IST-Lisbon database, https://fr.lxcat.net, (accessed 3 June 2019).

115 TRINITI database, https://fr.lxcat.net, (accessed 3 June 2019).

116 R. S. Berry, Radiat. Res., 1974, 59, 367-375.

117 S. Jo, D. H. Lee, K.-T. Kim, W. S. Kang and Y.-H. Song, Phys. Plasmas, 2014, 21, 103504.

118 S. Jo, D. H. Lee, W. S. Kang and Y.-H. Song, Phys. Plasmas, 2013, 20, 083509 .

119 S. Jo, D. Hoon Lee and Y.-H. Song, Chem. Eng. Sci., 2015, 130, 101-108.

120 W. Zhu, A. Inspektor, A. R. Badzian, T. McKenna and R. Messier, J. Appl. Phys., 1990, 68, 1489-1496.

121 R. K. Janev and D. Reiter, Phys. Plasmas, 2002, 9, 4071-4081.

122 R. K. Janev and D. Reiter, Phys. Plasmas, 2004, 11, 780-829.

123 L. S. Kassel, J. Am. Chem. Soc., 1932, 54, 3949-3961.

124 D. L. Baulch, C. J. Cobos, R. A. Cox, C. Esser, P. Frank, Th. Just, J. A. Kerr, M. J. Pilling, J. Troe, R. W. Walker and J. Warnatz, J. Phys. Chem. Ref. Data, 1992, 21, 411-734.

125 D. L. Baulch, C. J. Cobos, R. A. Cox, P. Frank, G. Hayman, Th. Just, J. A. Kerr, T. Murrells, M. J. Pilling, J. Troe, R. W. Walker and J. Warnatz, J. Phys. Chem. Ref. Data, 1994, 23, 847-848.

126 D. L. Baulch, C. T. Bowman, C. J. Cobos, R. A. Cox, Th. Just, J. A. Kerr, M. J. Pilling, D. Stocker, J. Troe, W. Tsang, R. W. Walker and J. Warnatz, J. Phys. Chem. Ref. Data, 2005, 34, 757-1397.

127 W. Tsang, J. Phys. Chem. Ref. Data, 1988, 17, 887-951.

128 W. Tsang, J. Phys. Chem. Ref. Data, 1991, 20, 221-273.

129 F. A. Lindemann, S. Arrhenius, I. Langmuir, N. R. Dhar, J. Perrin and W. C. M. Lewis, Trans. Faraday Soc., 1922, 17, 598-606.

130 J. Troe, Berichte Bunsenges. Für Phys. Chem., 1983, 87, 161169.

131 D. Smith and N. G. Adams, Int. J. Mass Spectrom. Ion Phys., 1977, 23, 123-135.

132 J. Woodall, M. Agúndez, A. J. Markwick-Kemper and T. J. Millar, Astron. Astrophys., 2007, 466, 1197-1204.

133 Y. Kim, Icarus, 1994, 112, 310-325.

134 In Collected Papers of L.D. Landau, Elsevier, 1965, pp. 63-66.

135 R. N. Schwartz, Z. I. Slawsky and K. F. Herzfeld, J. Chem. Phys., 1952, 20, 1591-1599.

136 E. E. Nikitin and J. Troe, Phys. Chem. Chem. Phys., 2008, 10, 1483-1501.

137 A. Lifshitz, J. Chem. Phys., 1974, 61, 2478-2479.

138 A. Starikovskiy and N. Aleksandrov, Prog. Energy Combust. Sci., 2013, 39, 61-110.

139 A. Hurlbatt, A. R. Gibson, S. Schröter, J. Bredin, A. P. S. Foote, P. Grondein, D. O'Connell and T. Gans, Plasma Process. Polym., 2017, 14, 1600138.

140 C. D. Pintassilgo, C. Jaoul, J. Loureiro, T. Belmonte and T. Czerwiec, J. Phys. Appl. Phys., 2007, 40, 3620-3632.

141 M. S. Benilov and G. V. Naidis, Int. J. Hydrog. Energy, 2006, 31, 769-774.

142 A. Ağıral, C. Trionfetti, L. Lefferts, K. Seshan and J. G. E. (Han) Gardeniers, Chem. Eng. Technol., 2008, 31, 1116-1123.

143 J. Luche, O. Aubry, A. Khacef and J.-M. Cormier, Chem. Eng. J., 2009, 149, 35-41. 
144 N. KHADIR, K. KHODJA and A. BELASRI, Plasma Sci. Technol., 2017, 19, 095502.

145 S. V. Kudryashov, A. Yu. Ryabov and A. N. Ochered'ko, High Energy Chem., 2018, 52, 167-170.

146 W. Wang, A. Berthelot, Q. Zhang and A. Bogaerts, J. Phys. Appl. Phys., 2018, 51, 204003.

147 P. Dordizadeh, K. Adamiak and G. S. P. Castle, Plasma Sources Sci. Technol., 2016, 25, 065009.

148 G. V. Naidis, Plasma Res. Express, 2018, 1, 017001.

149 M. Lisnyak, M. D. Cunha, J.-M. Bauchire and M. S. Benilov, J. Phys. Appl. Phys., 2017, 50, 315203.

150 G. Colonna and A. D'Angola, Plasma Modeling Methods and Applications, IOP Publishing, 2016.

151 G. V. Naidis, J. Phys. Appl. Phys., 2007, 40, 4525-4531.

152 M. Gao, Y. Zhang, H. Wang, B. Guo, Q. Zhang and A. Bogaerts, Catalysts, 2018, 8, 248.

153 F. Taccogna, J. Plasma Phys., , DOI:10.1017/S0022377814000567.

154 A. H. Khoja, M. Tahir and N. A. S. Amin, Energy Convers. Manag., 2019, 183, 529-560.

155 J. C. Whitehead, J. Phys. Appl. Phys., 2016, 49, 243001.

156 N. Chen, Y. Huang and A. L. Utz, J. Phys. Chem. A, 2013, 117, 6250-6255.

157 L. Chen, H. Ueta, R. Bisson and R. D. Beck, Faraday Discuss., 2012, 157, 285-295.

158 R. Bisson, M. Sacchi and R. D. Beck, Phys. Rev. B, 2010, 82, 121404.

159 D. R. Killelea, V. L. Campbell, N. S. Shuman and A. L. Utz, Science, 2008, 319, 790-793.

160 R. Bisson, M. Sacchi, T. T. Dang, B. Yoder, P. Maroni and R. D. Beck, J. Phys. Chem. A, 2007, 111, 12679-12683.

161 P. Maroni, D. C. Papageorgopoulos, M. Sacchi, T. T. Dang, R. D. Beck and T. R. Rizzo, Phys. Rev. Lett., 2005, 94, 246104.

162 L. B. F. Juurlink, R. R. Smith, D. R. Killelea and A. L. Utz, Phys. Rev. Lett., 2005, 94, 208303.

163 R. R. Smith, D. R. Killelea, D. F. DelSesto and A. L. Utz, Science, 2004, 304, 992-995.

164 R. D. Beck, P. Maroni, D. C. Papageorgopoulos, T. T. Dang, M. P. Schmid and T. R. Rizzo, Science, 2003, 302, 98-100.

165 M. P. Schmid, P. Maroni, R. D. Beck and T. R. Rizzo, J. Chem. Phys., 2002, 117, 8603-8606.

166 J. Higgins, A. Conjusteau, G. Scoles and S. L. Bernasek, J. Chem. Phys., 2001, 114, 5277-5283.

167 L. B. F. Juurlink, P. R. McCabe, R. R. Smith, C. L. DiCologero and A. L. Utz, Phys. Rev. Lett., 1999, 83, 868-871.

168 K. G. Prasanna, R. A. Olsen, Á. Valdés and G.-J. Kroes, Phys. Chem. Chem. Phys., 2010, 12, 7654-7661.

169 B. Jackson and S. Nave, J. Chem. Phys., 2011, 135, 114701.

170 B. Jackson and S. Nave, J. Chem. Phys., 2013, 138, 174705.

171 B. Jiang, R. Liu, J. Li, D. Xie, M. Yang and H. Guo, Chem. Sci., 2013, 4, 3249-3254.

172 B. Jiang and H. Guo, J. Phys. Chem. C, 2013, 117, 16127-16135.

173 M. Sacchi, D. J. Wales and S. J. Jenkins, J. Phys. Chem. C, 2011, 115, 21832-21842.

174 M. Sacchi, D. J. Wales and S. J. Jenkins, Phys. Chem. Chem. Phys., 2012, 14, 15879-15887.

175 B. Jiang, M. Yang, D. Xie and H. Guo, Chem. Soc. Rev., 2016, 45, 3621-3640.

176 E. C. Neyts and A. Bogaerts, J. Phys. Appl. Phys., 2014, 47, 224010.

177 W. Somers, A. Bogaerts, A. C. T. van Duin and E. C. Neyts, J. Phys. Chem. C, 2012, 116, 20958-20965.
178 W. Somers, A. Bogaerts, A. C. T. van Duin, S. Huygh, K.M.M. Bal and E. C. Neyts, Catal. Today, 2013, 211,01310-1136./DORE00024H

179 W. Somers, A. Bogaerts, A. C. T. van Duin and E. C. Neyts, Appl. Catal. B Environ., 2014, 154-155, 1-8.

180 M. Shirazi, E. C. Neyts and A. Bogaerts, Appl. Catal. B Environ., 2017, 205, 605-614.

181 T. Nozaki, H. Tsukijihara, W. Fukui and K. Okazaki, Energy Fuels, 2007, 21, 2525-2530.

182 T. Nozaki, T. Hiroyuki and K. Okazaki, Energy Fuels, 2006, 20, 339-345.

183 X. Zheng, S. Tan, L. Dong, S. Li and H. Chen, Chem. Eng. J., 2015, 265, 147-156.

184 X.-G. Zheng, S.-Y. Tan, L.-C. Dong, S.-B. Li, H.-M. Chen and S.-A. Wei, Fuel Process. Technol., 2015, 137, 250-258.

185 J. Kim, D. B. Go and J. C. Hicks, Phys. Chem. Chem. Phys., 2017, 19, 13010-13021.

186 Y. Mao, H.-F. Wang and P. Hu, WIREs Comput. Mol. Sci., 2017, 7, e1321.

187 P. N. Kechagiopoulos, J. W. Thybaut and G. B. Marin, Ind. Eng. Chem. Res., 2014, 53, 1825-1840.

188 K. M. Bal, S. Huygh, A. Bogaerts and E. C. Neyts, Plasma Sources Sci. Technol., 2018, 27, 024001. 